\title{
Analytical Rheology of Polymer Melts: State of the Art
}

\author{
Sachin Shanbhag \\ Department of Scientific Computing, Florida State University, Tallahassee, FL 32306, USA \\ Correspondence should be addressed to Sachin Shanbhag, sshanbhag@fsu.edu
}

Received 25 July 2012; Accepted 26 August 2012

Academic Editors: Y. Nakayama and A. O. Neto

Copyright () 2012 Sachin Shanbhag. This is an open access article distributed under the Creative Commons Attribution License, which permits unrestricted use, distribution, and reproduction in any medium, provided the original work is properly cited.

The extreme sensitivity of rheology to the microstructure of polymer melts has prompted the development of "analytical rheology," which seeks inferring the structure and composition of an unknown sample based on rheological measurements. Typically, this involves the inversion of a model, which may be mathematical, computational, or completely empirical. Despite the imperfect state of existing models, analytical rheology remains a practically useful enterprise. I review its successes and failures in inferring the molecular weight distribution of linear polymers and the branching content in branched polymers.

\section{Introduction}

Complex fluids, which include materials such as foams, gels, colloids, polymers, and emulsions, lie somewhere in the continuum between ideal solids and liquids. When subjected to an external deformation, they exhibit elasticity or memory-like classical solids; they also relax and dissipate energy by viscous flow-like classical liquids. This explains why these materials are called "viscoelastic." The systematic study of the flow behavior of such materials is called rheology [1-3]. More precisely, rheology is the relationship between stresses generated within a viscoelastic material, in response to an applied deformation.

1.1. Analytical Rheology. The rheology of many complex fluids depends very sensitively on the material microstructure. For example, the rheology of oil-water emulsions, which is important in food, cosmetic, and drug formulation industries, is determined by the concentration and particle size distribution [4-6]. This essential link between the microstructure and rheology provokes the idea of analytical (or analytic) rheology, which seeks to infer microstructural information from viscoelastic measurements. The primary motivation for analytical rheology, as elaborated more specifically later, is threefold: (i) many important microstructural features are extremely hard to probe using standard analytical techniques, (ii) viscoelastic measurements are often much more sensitive particularly to the microstructural features of most interest, and (iii) in the linear regime, rheology is cheaper and more convenient to measure.

In order to realize the promise of analytical rheology, we require a reasonably accurate rheological model, which may range from completely empirical models to those purely based on a molecular understanding of the underlying physics (see Figure 1). These models take, as their input, details of the material microstructure and the nature of the applied deformation and yield, as their output, a prediction of the rheological response. Analytical rheology, on the other hand, seeks to perform the inverse operation and hence is sometimes referred to in the literature as inverse rheology. Given the rheology of an unknown sample, it uses a rheological model, to provide details of the microstructure.

It should be pointed out that, while there is considerable overlap, the goal of analytical rheology is somewhat different from that of in silico design of polymeric materials [7-11]. Analytical rheology is a diagnostic tool, which is useful to determine the microstructure of a particular sample. The goal of in silico design is to determine what the microstructure should be, given a desired material response. Put differently, in "analytical" rheology, the analysis is performed after the measurement of rheology, while it is performed before experimental synthesis in in silico "design." Despite this subtle difference, they both employ similar material modeling and computational methods to achieve their goals, and there is substantial cross-talk between them. 


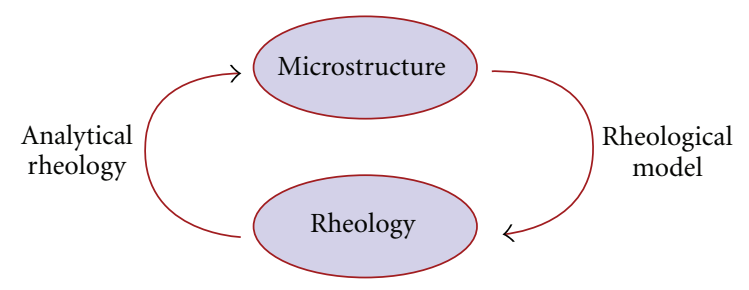

FIgURE 1: A rheological model takes microstructural information as input and provides the rheological response as output, while the goal of analytical rheology is the reverse. It is supplied with the rheological response of an unknown sample, and it provides details of the microstructure as output.

Recent advances in analytical rheology for all the different kinds of complex fluids are too broad a topic to cover adequately. Hence, in this paper, we will focus on a particularly important class of complex fluids, namely, polymer melts, which have seen tremendous progress in both rheological modeling and analytical rheology, over the past 30 years. In addition, purely in terms of commercial impact, polymers are among the most important members of the complex fluids family.

1.2. Polymers. Since the development of the first truly synthetic polymer, Bakelite, in 1909, synthetic polymers have come to pervade every aspect of our lives-ranging from automobiles to health-care, and from packaging to the consumer electronics industry [12]. Synthetic polymers are lightweight, corrosion resistant, easy to process into complex shapes, and possess properties that can optimize for specific applications by engineering the chemistry and spatial orientation of monomers.

Among synthetic polymers, polyolefins, which include polyethylene and polypropylene, constitute an important class. Together, they account for more than half of the synthetic polymer produced worldwide on a volume basis, with the current annual global production hovering around 100 billion kilograms. Our treatment in this paper will reflect a bias towards polyethylene and polypropylene due to their status as commercial juggernauts.

1.2.1. Architecture. While linear polymers, which consist of monomers chained together, are quite common, there is a rich diversity of polymer architectures, including ring and hyperbranched polymers (Figure 2). Theoretical understanding and industrial applications of synthetic ring polymers are currently underdeveloped. Hence, we will not cover them in this paper. When side branches are synthetically attached to a linear polymer (LP), we get branched or comblike polymers. Branched polymers (BPs) are of immense technological importance, especially for polyolefins, and have been studied extensively over the past 60 years [13-18]. Besides direct implications for processability, branching can also affect attributes such as tack and peel properties of pressure-sensitive adhesives [19]. Branching also plays an important physiological role in natural polymers such as starch, which is a polysaccharide consisting of glucose units

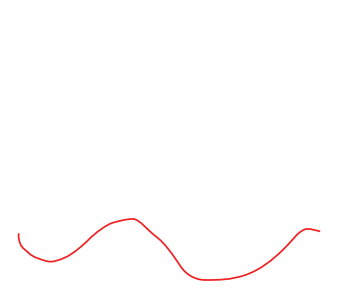

(a)

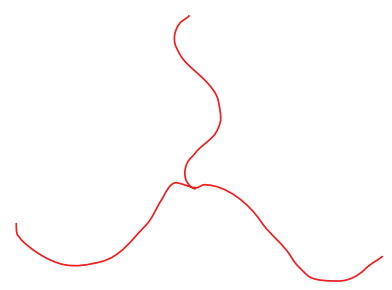

(b)

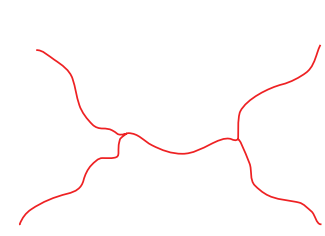

(c)

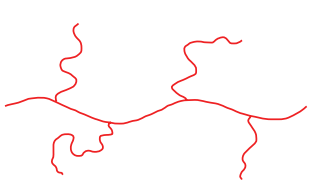

(e)

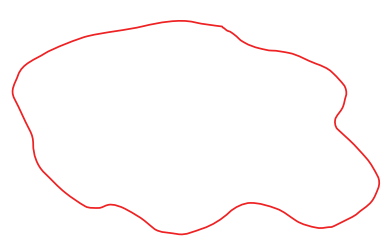

(d)

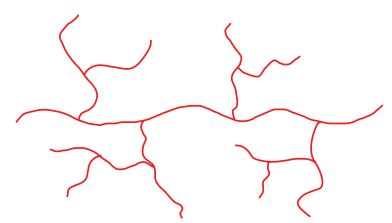

(f)
Figure 2: Different polymer architectures: (a) linear, (b) star, (c) H-polymer, (d) ring or cyclic, (e) comb, (f) hyperbranched.

chained together by glycosidic bonds, where it determines digestibility [20]. In highly branched structures, such as dendrimers and other hyperbranched polymers [21], branching enables applications in a variety of industries that include coatings, additives, drug and gene delivery, and nanotechnology.

When the length of the side arms is more than a few hundred carbon atoms (about 2-3 times the entanglement molecular weight $M_{e}$ ), the branching is classified as longchain branching (LCB) $[22,23]$. It is classified as short-chain branching $(\mathrm{SCB})$, when the arms are shorter.

Both SCB and LCB affect polymer properties, but the manner in which they do so is relatively independent of each other. Thus, the length and distribution of SCB can be tuned to control properties [24-32] such as crystallization kinetics, morphology, blend miscibility, mechanical properties of blown or compression molded films such as tear and impact strengths, and resistance to crack growth. Ultimate mechanical properties and crack resistance improve as the length of the side branch increases, possibly because SCB influences morphology and tie-molecule concentration, requiring greater energy to be absorbed to initiate cracks [33]. SCB is usually detected and quantified using analytical techniques like solution differential scanning calorimetry (DSC), temperature rising elution fractionation (TREF), and crystallization analysis fractionation (CRYSTAF) [34-39].

Compared to LCB, the effect of SCB on rheology is very weak [18-40]. On the other hand, the effect of LCB, on crystallization and ultimate mechanical properties, is relatively small. However, LCB has an enormous impact of rheological properties. It has long been known that the addition of trace 
amounts of long-chain branching (LCB) produces dramatic changes in the linear and nonlinear rheology [40-45]. These well-documented effects include a departure from the "3.4 power law" relating the zero shear viscosity $\eta_{0}$ to the weightaveraged molecular weight, unusually large sensitivity of $\eta_{0}$ to temperature or higher flow activation energies [46], enhanced shear thinning and strain hardening that lead to improved tear resistance, processability, and so forth.

What is the practical importance of LCB? Consider the illustrative example of isotactic polypropylene, which has many desirable solid-state properties such as high melting point, tensile strength, and stiffness. Unfortunately, it has poor melt strength, which seriously impairs its ability to be processed by foaming, blow molding, thermoforming, and so forth. Adding LCB fixes the melt strength. LCB may be introduced synthetically, by postreactor chemical modification [47-49], or by electron beam irradiation [50-52].

In an industrial setting, this dichotomy of SCB and LCB is helpful, since the levels of both types of branching can be independently adjusted to control rheology (important for melts during processing) and crystallinity (important for ultimate solid-state properties), independently.

1.3. Scope and Organization. For the most part, we will confine ourselves to the linear viscoelastic regime, which is the regime of small deformations. In this regime, the material obeys the principle of superposition. Our theoretical understanding of the physics, and ability to make quantitative predictions based on microscopic models are most mature in this linear regime. In addition, experiments in the linear viscoelastic regime (usually small angle oscillatory shear) are relatively easy to perform, which makes it an ideal candidate for analytical rheology. Nonlinear rheology, which depends on both the deformation history and the flow type, is sometimes far more sensitive to some features of molecular structure, such as LCB. However, theoretical developments in this regime have not fully matured, partly due to the difficulty in obtaining high-quality data in the strongly nonlinear regime [53].

I will review the specific advantages of analytical rheology of LPs and BPs in the next section. I will then present a brief summary of different molecular models used to study polymer dynamics and rheology and argue why the tube model is an attractive candidate as the rheological model to invert. For LPs, I will focus on the inversion of linear viscoelastic spectra to reconstruct the molecular weight distribution and point out the different techniques and challenges. For BPs, I will review different experimental and modeldriven methods to infer the level of LCB from linear and nonlinear viscoelastic measurements. This material is covered in Section 4.

\section{Motivation and Background}

As described next, the primary motivation for analytical rheology of LPs is convenience, cost, and resolution. For BPs, analytical rheology is often a necessity-a technique of last resort-due to the lack of alternatives.
2.1. Linear Polymers. The problem of analytical rheology for LPs has a relatively straightforward mathematical description: given the linear viscoelastic response of a mixture of LPs, typically in the form of oscillatory shear measurements, determine the molecular weight distribution. In the most general case, the mixture may be both multimodal (having multiple peaks) and polydisperse (having broad distributions). In the literature, terms like "bidisperse" are commonly used to mean "bimodal." For clarity, we will avoid this terminology. It is quite natural to ask, given the availability of techniques such as size-exclusion chromatography (SEC) and light scattering, "what does analytical rheology offer that these other techniques do not?"

All of these traditional analytical methods for determining molecular weight distribution require dissolving the polymer in a solvent, ideally at room temperature, which can be tricky for many industrially relevant polymers such as polyethylene, polypropylene, Teflon, and others [54-59]. Rheological measurements circumvent this nontrivial and time-consuming dissolution step and are indeed straightforward to perform on essentially all commercially important polymers.

A second important factor in favor of rheology is its sensitivity to the high molecular weight tail of the distribution, which is often important to characterize accurately, because of its outsized influence on processability. The sensitivity of rheology to molecular weight is unparalleled. For example, doubling the molecular weight of a moderately entangled LP causes the zero-shear viscosity to increase approximately 10fold. For a similar doubling of the arm-molecular weight of a symmetric star polymer, the zero-shear viscosity increases a dramatic 1000-fold. In contrast, the molecular weight dependence of SEC $\left(\sim M^{0.5}\right)$ and light scattering $\left(\sim M^{1}\right)$ are much weaker. The dependence of other linear and nonlinear material functions on molecular weight is also quite pronounced. This strong dependence can, in principle, be directly translated into analytical sensitivity and resolution, especially at higher molecular weights where competing analytical methods are particularly ill-suited $[54,60,61]$.

From an industrial standpoint, perhaps no other driving force is as important as cost. Here too, rheology has an important advantage over chromatography; it typically costs only about a third of the cost of SEC [62]. In addition to these obvious advantages, carrying out routine linear viscoelastic measurements has simultaneously become more routine and accurate, enabling nonexperts to perform experiments competently. Consequently, analytical rheology of LPs has made its way into some commercial rheometers.

2.2. Branched Polymers. Characterization of BPs with analytical rheology inherits all of the advantages listed above for LPs, such as the elimination of the solvation step and lower energy costs. In addition, the sensitivity of rheology to the molecular weight of LPs is replaced by an even greater sensitivity to the presence of LCB.

The nature and level of LCB in polymers depends on their synthesis and postsynthesis treatment. Consider, for example, low-density polyethylene (LDPE) which is synthesized 
using radical chemistry and has about $0.5-4.0 \mathrm{LCB} / 1000$ $\mathrm{C}$ atoms, and high-density polyethylene (HDPE) which is synthesized using Ziegler Natta, Philips, or metallocene catalysts and approximately has $0.1-0.2 \mathrm{LCB} / 1000 \mathrm{C}$ atoms in the backbone [63]. LDPE is highly branched, with significant amounts of both SCB and LCB, while metallocene HDPEs can have very well-controlled and predictable amounts of LCB and SCB.

Thus, today, breakthroughs in catalyst technology have allowed us to produce industrial quantities of polymers with precisely controlled molecular structure [64, 65]. However, these advances in chemistry have not been accompanied by simultaneous advances in the characterization of LCB. We are left with a situation where the resolution of standard analytical tools available for diagnosing LCB is much lower than our ability to synthesize sparse levels of precisely controlled branching. The need to develop analytical methods to accurately detect and quantify these trace levels of LCB has become increasingly urgent, and analytical rheology has emerged as a particularly promising candidate [66].

Historically, three categories of methods have been used to diagnose LCB in synthetic polymers: (i) $g$-ratio analysis, (ii) spectroscopy, and (iii) rheology.

The presence of branch points causes conformational change in BPs, often causing them to shrink relative to the size of LPs. The " $g$-ratio" in $g$-ratio analysis is the ratio of the size of the branched and LPs of identical molecular weight. Theoretical estimates of $g$ for different polymer architectures are available via Zimm-Stockmayer equations $[67,68]$. For example, the $g$-ratio of a star molecule, $g_{\text {star }}=(3 f-2) / f^{2}$, where $f$ is the number of branches. Similarly, for randomly branched comb polymers with backbones and branches of constant size, Casassa and Berry [69] obtained

$g_{\mathrm{comb}}=\frac{1}{(1+\rho f)^{3}}\left[1+2 f \rho+\left(2 f+f^{2}\right) \rho^{2}+\left(3 f^{2}-2 f\right) \rho^{3}\right]$,

where $\rho$ is the ratio of the molecular weight of the branch and the backbone.

More accurate results are obtained from Monte Carlo simulations and other numerical techniques [70-76]. The principal idea is to measure the size and molecular weight of a BP using experimental techniques such as SEC, light scattering, or intrinsic viscosity (Mark-Houwink plots), and so forth, and then use the available $g$-ratios to determine the nature and degree of branching. The use of $g$-ratio analysis in isolation to detect LCB is risky, because trace levels of LCB result in only a modest change (see Figure 3 ) in size [66, 77]. However, it is a useful tool when used in conjunction with more sophisticated multidetector methods $[40,78,79]$, as described later.

${ }^{13} \mathrm{C}$ nuclear magnetic resonance (NMR) is a spectroscopic method that can be used to directly probe LCB $[80-$ 85 ], by detecting tertiary $\mathrm{C}$-atoms linking the branch to the backbone. Indeed, it is widely used as a gold standard against which the accuracy and sensitivity of other techniques are measured. However, ${ }^{13} \mathrm{C}$ NMR is a questionable gold standard at best and suffers from several technical limitations. For example, NMR is unable to differentiate between

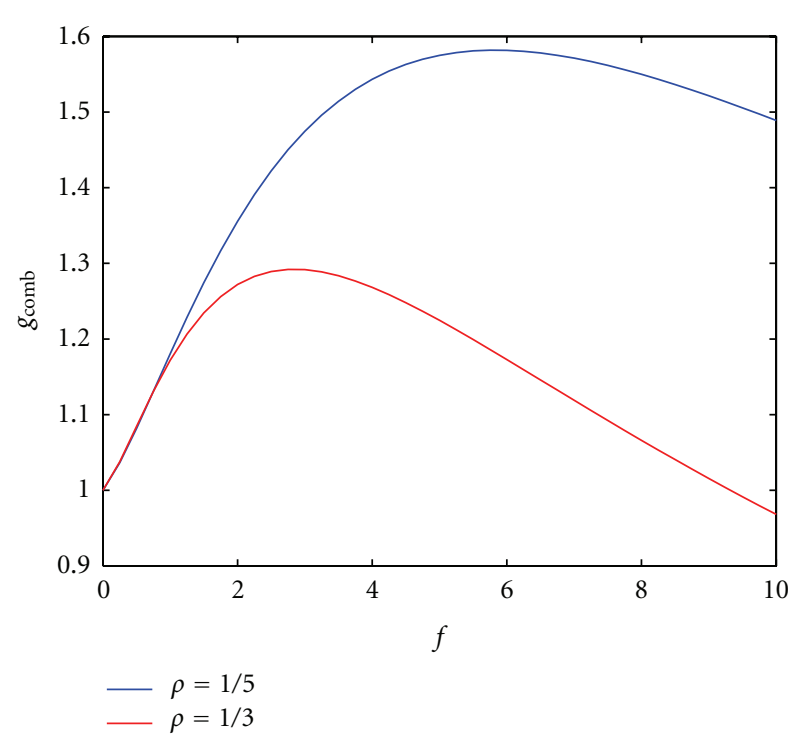

FIGURE 3: $g$-ratio of comb polymers computed from (1) for two different values of $\rho$. For small values of $f, g_{\text {comb }}$ is not strong function of the branching level.

short and long branches. In particular, it cannot distinguish between branches with more than 10 carbon atoms in the side chain. This length is far below the threshold of around 100-200 carbon atoms in "long-" chain branching [42, 8691].

In summary, both $g$-ratio analysis and NMR are tedious and expensive and require specialized training in carrying out the necessary experiments. However, their most important drawback is that they probe fundamentally weak signals. Since the actual number of branch points is often a very small fraction of the total number of backbone carbons, the signal-to-noise ratio can become a serious issue $[66,92]$. This opens up a natural niche for analytical rheology as molecular dynamics are extremely sensitive to molecular structure, and small changes in LCB levels result in major changes in rheology [93].

2.3. Model-Driven Analytical Rheology. Although analytical rheology requires reasonably accurate rheological models, they need not be perfect. This can be explained schematically using Figure 4. The extreme sensitivity of rheology on molecular structure can be conceptually visualized as a straight line with a large slope: small changes in the structure are manifested as large changes in the rheological response. The inverse problem of analytical rheology is visualized in this schematic as finding the "structure" corresponding to a given measured "rheology." Since rheological models are not perfect, they are characterized by uncertainty which is depicted by the dotted lines parallel to the "true" model (red line). When the slope of the line is large, the inverse solution, even in the presence of uncertainty, is relatively tightly bracketed. Thus, the inversion of imperfect models in such situations can be practically meaningful. In contrast, if the structurerheology dependence were more modest, the slope of the line 

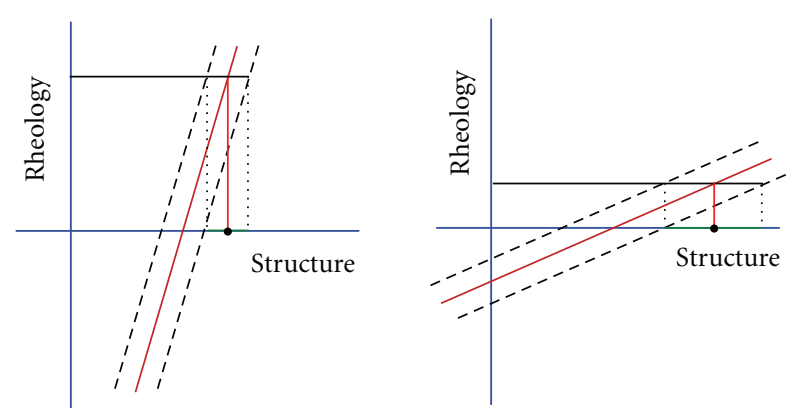

FIGURE 4: Since rheology is very sensitive to structure (left subfigure), the inverse problem of analytical rheology is easier to "solve" (see text) even with rheological models that are less than perfect (dotted lines). The same is not true, when rheology is not as sensitive to structure.

in the conceptual diagram would be smaller. Given models of comparable reliability, we would not be able to bracket the "true" structure with as much confidence. This is a key insight. The premise of model-driven analytical rheology relies on the strong dependence of structure and rheology to make up for the limitations of the rheological models themselves.

\section{Models for Polymer Dynamics and Rheology}

In this section, we present a historical record of the modeling of polymer melt rheology before considering the most popular ansatz, namely, the tube model, more closely. The tube model and its modifications are ideally suited for inversion using analytical rheology because they offer a reasonable mix of accuracy and efficiency. We will end this section with a brief remark about other more accurate, but computationally intensive, methods for modeling polymer dynamics and rheology. In the future, with faster computers and better algorithms, it is conceivable that some of these models could serve as the forward model.

3.1. Historical Development. Rubber elasticity theory considered the number of configurations available to Gaussian strands constrained between permanent chemical crosslinks to successfully describe the relationship between the stresses generated in a rubber-like material subjected to affine deformation [94-101]. To account for the transient nature of polymer entanglements, Green and Tobolsky generalized the rubber elasticity theory by visualizing entanglements as physical cross-links that were continuously broken and reformed [98-100, 102-104]. The temporary network model with a single time constant is the simplest model for polymer melts.

While the network theory for such rubber-like liquids was being advanced by Lodge and Yamamoto [105-110], progress was simultaneously being made in molecular modeling on the other end of the concentration spectrum, namely, dilute polymer solutions. Starting with Kuhn, who described the configuration of chains using a random coil model, landmark contributions were made, which suggested the importance of the chain-like structure of polymers [111117]. It was recognized that the connectivity of monomer units in a polymer chain should be directly represented in the molecular models and constitutive equations. Simultaneous advances in experiments and theory led to the discovery of universality: at time scales larger than those corresponding to motion of individual chemical bonds, dynamical properties of polymer chains of different chemical structures were similar. This observation greatly facilitated the development of coarse-grained descriptions of polymer dynamics, which continue to be popular to this day.

Rouse exploited the Gaussian character of polymer coils and developed the famous "Rouse model" by representing a polymer in dilute solution as a series of beads attached to its neighbors by springs sloshing around by thermal fluctuations in a viscous solvent [113]. The viscous drag was borne by the beads, and the elasticity was contributed by the springs. Interestingly, the Rouse model turned out to be a pretty poor model for dilute polymers-its intended target. However, it was found to be an excellent model for unentangled polymer melts, where its neglect of hydrodynamic coupling between different polymers was justified [118124]. Zimm improved upon the Rouse model by including hydrodynamic interactions which led to a better quantitative understanding [114].

For long linear chains in melts and concentrated solutions, the zero shear viscosity $\eta_{0} \sim M^{3.5 \pm 0.2}$, where $M$ is the molecular weight of the linear chain, whereas in dilute solutions the exponent was much closer to unity. To explain the much stronger dependence of rheology on structure, decorations in the form of elastic coupling of the submolecules and enhanced friction due to surrounding chains were added to the Rouse model $[125,126]$. However, the modifications were ad hoc and lost their connection to the molecular picture. The gridlock was broken after nearly two decades, when de Gennes [127] proposed the famous reptation model for a linear chain trapped in a permanent network. He suggested that the "test" chain could renew its configuration by slithering back and forth along the direction of the chain backbone like a snake.

This reptation or "tube" picture (shortly described in greater detail) was further developed by Doi and Edwards [128-131] to concentrated polymer solutions, which enabled the computation of various dynamical properties and eventually led to the famous Doi-Edwards constitutive equation for linear chains. In branched molecules reptation is suppressed because the branch point anchors the molecule in the middle. Pearson and Helfand [132] considered the retraction of a side branch in the tube to describe linear viscoelastic data. These seminal efforts were modified to include the evolution of the tube itself which is produced by the relaxation of matrix chains. This accelerating effect in real polymer melts was captured by the application of the approximate double reptation model for linear chains [133-135] and the dynamic dilution theory by Ball and McLeish [136] for BPs.

3.2. The Tube Model. de Gennes offered a phenomenological model to account for the slowdown in the molecular motion 
of linear chains in a melt for $M \gg M_{e}$, where $M_{e}$ is the molecular weight between entanglements. Originally, he considered the motion of a long polymer chain in a fixed or cross-linked rubber network [127]. The analysis could then be generalized to describe the dynamics of a chain moving in a mesh formed by surrounding chains instead of the fixed network. Such a "probe" chain, submerged in a sea of polymers, could be thought of as being confined to a hypothetical "tube." The tube permits the motion of the chain to be along the contour of the chain, while limiting its motion in the lateral direction to length scales of the order of the diameter of the tube (see Figure 5).

For an entangled system of polymer molecules in the linear viscoelastic regime, the chains relax their configurations by three mechanisms (i) reptation, (ii) contour length fluctuations, and (iii) constraint release.

Reptation is a method of stress relaxation that involves the curvilinear diffusion of a test chain along the axis of its tube, due to the uncrossability of topological constraints that the tube represents. Portions of the chain crinkle up within the tube creating slack or density fluctuations which diffuse through the tube and get released at the ends. When a linear molecule "snakes" its way out in this manner, it vacates portions of the original tube and relaxes the stress linked with those portions. After a system is subjected to an initial strain, the isotropic equilibrium distribution of chains is disturbed and the associated anisotropy generates stress. As parts of the chain project out of the original tube, they seek new tubes (get reentangled with other matrix chains) which unlike the original tube are isotropic and hence are not related with any stress. The unrelaxed stress at any time, therefore, is proportional to the fraction of the original tube that remains unvacated [104].

The second mechanism of stress relaxation is called contour-length or primitive-path fluctuations. Star polymers cannot reptate, because the branch point anchors the molecule down. The chain end of a star arm has to execute large entropically unfavorable primitive path fluctuations towards the tether point and then "breathe out" to explore a new tube. Just as in reptation, when the molecule reexpands into a new tube, the stress linked with the original tube is relaxed. Pearson and Helfand [132] considered the steep entropic barrier faced by a star arm attempting to retract towards the branching point. In contrast to linear chains in which the relaxation time, $\tau \sim M^{3.5 \pm 0.2}$, it has been shown for star arms $[132,137]$ that the relaxation time exhibits an exponential dependence on the arm molecular weight, $\tau \sim \exp \left(\right.$ constant $\left.\times M_{a}\right)$. Primitive-path fluctuations are a dominant mode of relaxation in polymers with more complicated branching architecture like $\mathrm{H}$-polymers and combs. The relaxation of the backbone of a $\mathrm{H}$-polymer occurs after the arms have completely relaxed by a retraction process similar to that described above for star arms.

As a probe chain reptates in its tube, the surrounding chains which form the tube also reptate in their own tubes. When a portion of a matrix chain slips past the probe chain, it releases the topological constraint it hitherto imposed on the probe chain and the latter is then free to

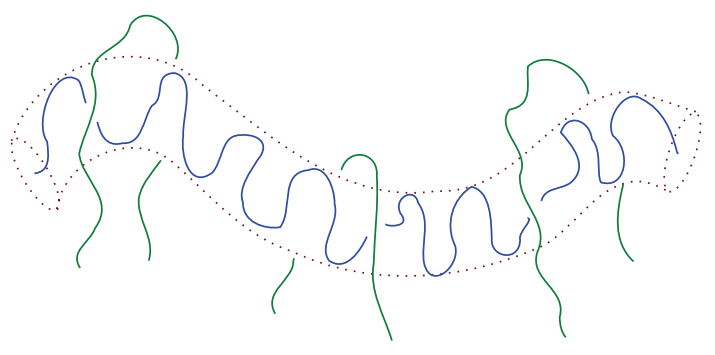

FIGURE 5: A schematic diagram depicting the origin of a hypothetical tube around a test chain due to the presence of matrix chains in a polymer melt.

reorient [138-140]. The movement of the matrix chains thus induces motion of the tube confining the probe chain, which, in turn, allows large scale motion of the probe chain in the lateral direction. The increased latitude for lateral motion can also be viewed as an increase in the effective diameter of the tube around the test chain. This increase in tube diameter due to simultaneous relaxation of matrix chains is incorporated into the tube model for BPs by using the notion of dynamic tube dilution [136].

3.2.1. Hierarchical Models. These three concurrent molecular processes can be weaved into a self-consistent mathematical framework to build computational models [93, 141-151]. The framework and models are too complicated and numerous to describe in detail here, but it is useful to point out the idea of hierarchical relaxation which, besides the "tube," is central to all these models.

For complicated branched architectures, it has been postulated and found from computer simulations [152, 153] that stress relaxation proceeds in a "hierarchical" manner. The tips of the outer-most branches are the first to relax via contour-length fluctuations. Once they have completely relaxed, they are modeled as viscous drag elements on the branches (or backbone) to which they are attached, which in turn commence relaxing. This process continues until the stress associated with the inner-most parts of the polymer is completely relaxed. These hierachical models are able to predict the linear viscoelastic response of arbitrary mixtures of polymers with arbitrary architectures in melts and have become increasingly accurate $[17,104,136,141-144,146$, 154-157], although numerous shortcomings exist and continue to be exposed [158-161]. The objective of these hierachical models is the prediction of complex modulus, $G^{*}(\omega)=G^{\prime}(\omega)+i G^{\prime \prime}(\omega)$, from structural data obtained using light-scattering, SEC, and NMR, using a small set of parameters that are determined from the chemistry of the polymer, and the temperature at which the measurement is carried out. Typically these include the density, equilibration time $\tau_{e}$, entanglement molecular weight $M_{e}$, and sometimes the plateau modulus, $G_{N}^{0}$ [162]. The same parameter values are used for different architectures with the same underlying chemistry. 
3.3. State of the Art. Enormous progress has been made in modeling the linear and nonlinear rheology of LPs using the tube model. Likhtman and McLeish [142] rigorously integrated all known processes of relaxation in the linear regime (reptation, contour length fluctuations, constraint release, longitudinal stress relaxation along the tube) simultaneously. Similarly, Graham et al. refined the original Doi and Edwards model to predict the rheological response of linear chains over the entire range of deformation rates from the linear to strongly nonlinear [155]. These include the additional effects of chain stretch and convective constraint release that are not present in the linear regime.

For BPs, the hierarchical models described above represent the state of the art in the linear viscoelastic regime. Progress in the nonlinear rheology of BPs has been less forthcoming. McLeish and Larson [53] developed a molecular constitutive model for a BP with arbitrary number of arms, but only two branch points. This "pom-pom model" was able to qualitatively describe strain hardening in extensional flows and strain softening in shear flows for the first time. Subsequent modifications have introduced drag-strain coupling [163], corrections for reversing flows [164], and zero second normal stress coefficient [165]. Inkson et al. [166] extended the pom-pom model to a multimode model, phenomenologically, and found that it described the nonlinear rheology of commercial LDPE quite well.

3.4. Computational Models. In addition to the tube model, there are a number of other simulation methods, such as sliplink [167-178], lattice [179-191], and molecular dynamics [192-205] models, to model polymer dynamics. These methods are more "accurate" since they avoid the central simplification of the mean-field tube theory, namely, the phenomenological approximation of the effects of a complex multi-chain environment into a mean-field "tube." While this results in an analytically tractable single-body problem, it buries assumptions that have to be made about multibody interactions such as the phenomenon of constraint release, the coupling of entanglements in a melt, and the form of the mean-field potential. Without doubt these assumptions sometimes lead to cancellation of errors, but not always, and certainly not in any predictable fashion.

It should be pointed out that increased accuracy comes at the price of increased computational cost. A significant problem in modeling polymers is the wide separation of dynamical timescales, ranging from the order of picoseconds $\left(10^{-15}-10^{-9} \mathrm{~s}\right)$ for local bond vibrations, to the order of seconds or hours $\left(10^{-3}-10^{3} \mathrm{~s}\right)$ for phenomena of greatest relevance to rheology. A possible solution is coarse-graining, where fast dynamics are averaged out, and only slow modes of motion are explicitly accounted for. Thus, slip link models are coarse-grained at a length scale of the order $\sim 10$ nanometers, and a time scale of the order 10-100 nanoseconds. Similarly lattice and molecular dynamics models are coarsegrained at the level of an effective monomer, which roughly corresponds to a length scale of about a nanometer. Despite the increase in computational power in recent years and the possibility of coarse-graining, these models are not yet fast enough to be useful for analytical rheology.

\section{Methods and Progress}

In this section, I will review developments in analytical rheology of LPs and BPs. Advances have been more forthcoming for polydisperse mixtures of LPs for three reasons, (i) there is a plethora of well-characterized experimental data $[60,206-212]$, (ii) rheological models have been built, tested, refined, and calibrated extensively [213], and (iii) it is possible to use approximate "double reptation-" type models $[135,214]$, in lieu of the full tube model [142], which makes the mathematical model and its numerical solution more expedient.

Numerous attempts have been made at analytical rheology of BPs, especially for diagnosing branching content with varying degrees of success. The primary impediments are that (i) synthesizing well-defined and well-characterized BPs is highly nontrivial [7, 215-221], (ii) the process of testing and calibrating the rheological models exposes many weaknesses of the tube model that are not manifested for LPs $[158,159]$, and (iii) there is no counterpart to the "double reptation" model because of the complexity of the computational models. Even so, the past few years have seen tremendous progress.

4.1. Linear Polymers. A particularly influential idea in the development of analytical rheology for LPs was "double reptation," which offered a mathematically convenient approximation with empirical support $[54,222]$, to a more complete but complex theory $[135,142,214]$. It postulated that the relaxation modulus $G(t)$ was related to the molecular weight distribution $w(M)$ via the convolution:

$$
\frac{G(t)}{G_{N}^{0}}=\left(\int_{M_{e}}^{\infty} F^{1 / \beta}(M, t) w(M) d \ln M\right)^{\beta},
$$

where $M_{e}$ is the entanglement molecular weight, the kernel $F(M, t)$ is the relaxation function of a monodisperse LP, and $G_{N}^{0}$ is the plateau modulus. If $W(M)$ is the weight fraction of the polymers with molecular weight less than $M$, then the molecular weight distribution is defined as $w(M)=$ $d W(M) / d \ln M . \beta$ is the mixing exponent which is 1 for single reptation in the original "reptation in a fixed tube" model [104], and 2 in the original double reptation model. The double reptation model originates from the idea of entanglements as binary contacts [62], which, by itself, is still an unsettled issue $[189,223-228]$. The restriction on the value of $\beta$ can be relaxed on numerical and physical grounds, allowing any value of $\beta \geq 1$. Depending upon how the experimental data is parsed, values of $\beta$ ranging from slightly below 2 to nearly 4 have been used [229-231].

A number of plausible forms, ranging from purely theoretical to completely empirical, have been proposed for the kernel $F(M, t)$. The more complex empirically inspired 
kernels usually introduce additional model/material dependent parameters. Some popular choices, roughly sorted in the order of increasing complexity, include the following.

(i) The Tuminello or step function kernel [133]:

$$
F^{1 / \beta}(M, t)= \begin{cases}1 & \text { if } t<\tau(M) \\ 0 & \text { otherwise }\end{cases}
$$

where $\tau(M)$ is the terminal relaxation time, which is often set to $\tau(M)=K(T) M^{\alpha}$, where $\alpha \approx 3.4$, and $K(T)$ is a function of temperature that encodes time-temperature superposition using, for example, Williams-Landel-Ferry or WLF parameters [232].

(ii) Single Exponential Form [135]:

$$
F^{1 / \beta}(M, t)=\exp \left(-\frac{t}{\beta \tau(M)}\right) .
$$

It smoothens out the discontinuity in the Tuminello kernel at $t=\tau(M)$.

(iii) The Doi-Edwards or reptation kernel [104]:

$$
F^{1 / \beta}(M, t)=\frac{8}{\pi^{2}} \sum_{n=\text { odd }}^{\infty} \frac{1}{n^{2}} \exp \left(-n^{2} \frac{t}{\tau(M)}\right) .
$$

It is derived from the original reptation model without contour-length fluctuations or constraint release.

(iv) The BSW (Baumgaertel, Schausberger, and Winter) kernel [233]:

$$
F^{1 / \beta}(M, t)=\alpha \int_{0}^{1} u^{\alpha-1} \exp \left(-\frac{t}{u \tau(M)}\right) d u,
$$

where $\alpha$ is a material parameter of the order of 0.5 , which depends on $G_{N}^{0}$ and $J_{e}^{0}$.

(v) The time-dependent diffusion de Cloizeaux model [134]:

$$
F^{1 / \beta}(M, t)=\frac{8}{\pi^{2}} \sum_{n=\text { odd }}^{\infty} \frac{1}{n^{2}} \exp \left(-n^{2} U(t, M)\right),
$$

with

$$
U(t, M)=\frac{t}{\tau(M)}+\frac{M^{*}}{M} g\left(\frac{M t}{M^{*} \tau(M)}\right),
$$

where $g(x) \approx-x+\sqrt{x} \sqrt{x+\sqrt{\pi x}+\pi}$, and $M^{*}$ is a parameter.

Typically, the experimental $G^{*}(\omega)$ is available at a set of $m$ discrete experimental frequencies as $\left\{\omega_{i}, G_{\exp }^{\prime}\left(\omega_{i}\right), G_{\text {exp }}^{\prime \prime}\left(\omega_{i}\right)\right\}$, where $i=1,2, \ldots m$. The idea is to minimize the function,

$$
\begin{aligned}
\chi^{2}=\frac{1}{2 m} \sum_{i=1}^{m}[ & \left(\frac{G_{\text {exp }}^{\prime}\left(\omega_{i}\right)-G_{\mathrm{th}}^{\prime}\left(\omega_{i}\right)}{G_{\text {exp }}^{\prime}\left(\omega_{i}\right)}\right)^{2} \\
& \left.+\left(\frac{G_{\text {exp }}^{\prime \prime}\left(\omega_{i}\right)-G_{\mathrm{th}}^{\prime \prime}\left(\omega_{i}\right)}{G_{\text {exp }}^{\prime \prime}\left(\omega_{i}\right)}\right)^{2}\right],
\end{aligned}
$$

where the subscript "th" refers to the theoretical dynamic moduli corresponding to the relaxation modulus $G(t)$ in (2).

We can translate between $G(t)$ and $G^{*}(\omega)$ either by using Schwarzl approximations $[231,234]$ or by extracting the underlying relaxation spectrum $h(\tau)[62,229,235,236]$. The relationships between $G(t), G^{*}(\omega)$, and $h(\tau)$ are given by

$$
\begin{aligned}
& G^{\prime}(\omega)=\int_{-\infty}^{\infty} \frac{\omega^{2} \tau^{2}}{1+\omega^{2} \tau^{2}} h(\tau) d \ln \tau, \\
& G^{\prime \prime}(\omega)=\int_{-\infty}^{\infty} \frac{\omega \tau}{1+\omega^{2} \tau^{2}} h(\tau) d \ln \tau, \\
& \frac{G(t)}{G_{0}}=\int_{-\infty}^{\infty} h(\tau) \exp \left(-\frac{t}{\tau}\right) d \ln \tau .
\end{aligned}
$$

The extraction of $h(\tau)$ from $G^{*}(\omega)$ is a celebrated inverse problem, with its own chequered past [18, 237-254].

Once a choice of the kernel $F(M, t)$ and the method from translating between $G(t)$ and $G^{*}(\omega)$ are made, the process of inferring $w(M)$ from (1) essentially involves "solving" (2). This is also an inverse problem and numerous attempts have been made at figuring out optimal inversion strategies. Broadly, three categories of strategies can be identified.

(i) Regularization. Extraction of $h(\tau)$ in (10) and $w(M)$ in (2) involves inverting Fredholm integrals of the first kind. Regularization is a well-known strategy of addressing the illposedness of such problems [255]. In Tikhonov regularization [256], a smoothness criterion is applied on $w(M)$ by modifying $\chi^{2}$ in (9), to address the multiplicity of $w(M)$ consistent with a measured $G^{*}(\omega)$ due to the inherent susceptibility of the inverse problem to perturbations in the data [229, 230, 257-259]. More precisely, the numerical solution of (2) involves the discretization of the function $w(M)$ into the arrays $\left[w_{i}, M_{i}\right]$, with $1 \leq i \leq N$, and the time variable as $t_{j}$ as

$$
\left(\frac{G\left(t_{j}\right)}{G_{N}^{0}}\right)^{1 / \beta}=\sum_{i=1}^{N} F^{1 / \beta}\left(M_{i}, t_{j}\right) w_{i} .
$$

This equation can be thought of as a linear regression problem $A x \approx b$ with $A_{j, i}=F^{1 / \beta}\left(M_{i}, t_{j}\right), x_{i}=w_{i}$, and $b_{j}$ equal to the left-hand side of the above equation. In the classical linear least squares method, the residual $\|A x-b\|^{2}$ is minimized by solving the normal equations $A^{T} A x=A^{T} b$. However, if the matrix $A$ is ill-conditioned, as is often the case in these problems, Tikhonov regularization adds a regularization term to prefer solutions with desirable properties and seeks a solution that minimizes $\|A x-b\|^{2}+\|\Gamma x\|^{2}$, where $\Gamma$ is the Tikhonov matrix. The normal equations are modified to $\left(A^{T} A+\Gamma^{T} \Gamma\right) x=A^{T} b$ [256]. If the matrix $\Gamma$ is set to $\Gamma_{i, j}=\delta_{i, j}$, where $\delta_{i, j}$ is the Dirac delta function, then the regularization prefers solutions with small norms. It can be tailored to prefer solutions with small second derivatives (or smoother $w(M)$ ) by setting $\Gamma_{i, j}$ proportional to $\delta_{i, j}-$ $2 \delta_{i+1, j}+\delta_{i+2, j}$. The strength of the smoothness criterion can be controlled, and it is found that the inferred molecular weight distribution is somewhat sensitive to the strength 
of regularization [257]. Using similar methods, Maier et al. [229] extracted the kernel function from empirical data and found that it did not match any of the standard kernels satisfactorily.

(ii) Parameterization. In this strategy, a parametric form is assumed for $w(M)$, often by using prior knowledge of the polymerization process. For example, log-normal $w(M)$ is typical of Ziegler-Natta or addition polymers, while Schultz distributions are typical of condensation or metallocene polymers, Flory [16]. If the sample is suspected to be monomodal, then a generalized form such as three parameter generalized exponential (GEX) can be used [231, 260], which can capture the features of Schultz or log-normal distributions at the cost of an extra free parameter.

$$
w_{\mathrm{GEX}}(M) \sim\left(\frac{M}{M_{0}}\right)^{a+1} \exp \left(-\left[\frac{M}{M_{0}}\right]^{b}\right),
$$

where $a, b$, and $M_{0}$ are the three parameters. For more complex multimodal $w(M)$, this form can be extended to include a sum of generalized exponential models [231, 261, 262].

Pattamaprom and Larson used the dual-constraint model to modify the kernel $F(t, M)$ and parametrically deduced the $w(M)$ of multimodal and polydisperse LPs [263]. The dual constraint model can be used to predict the linear viscoelasticity of linears and stars and hence offers the possibility of treating BPs in a similar manner [264].

(iii) Stabilization. This strategy differs from the two above in significant ways. The philosophical difference stems from the fact that in practice we often do not seek the complete distribution $w(M)$, but only its principal moments [62, 235, $236,265]$. For example, we can define the $p$ th moment of $w(M)$ as

$$
\mu_{p}(w)=\int_{0}^{\infty} M^{p} w(M) d M
$$

and realize that the usual number-averaged molecular weight, $M_{n}=\mu_{1}(w)$, and the weight-averaged molecular weight, $M_{w}=\mu_{2}(w)$, and so forth. In the same spirit, one can consider moments of $G(t)$ as

$$
\mu_{q}(G)=\int_{0}^{\infty} t^{q} G(t) d t
$$

and realize that the zero-shear viscosity $\eta_{0}=\mu_{0}(G)$, the recoverable compliance $J_{e}^{0}=\mu_{1}(G) / \eta_{0}^{2}$, and so forth. In general, $p$ and $q$ need not be integers.

Using the idea of Mellin transforms [62, 266, 267], Mead established a connection between the moments $\mu_{p}(w)$, which tell us something about the molecular weight distribution, and the moments $\mu_{q}(G)$, which tell us something about the rheology. In particular, for a monomodal $w(M)$ with the single exponential form for the mixing rule or kernel $F(M, t)$ and $\tau(M)=K M^{\alpha}$, it can be shown that $[62,235]$ :

$$
\mu_{p}(w)=\left[\frac{1}{\Gamma(p / \alpha)(K \beta)^{p / \alpha}}\right] \mu_{((p / \alpha)-1)}(G),
$$

where $\Gamma$ is the standard gamma function. Thus, computing a particular moment of $w(M)$ involves extracting $G(t)$ from the experimental data, taking the appropriate moment, and using (16). For more complicated kernels and multimodal $w(M)$, it is usually not possible to write analytical expressions similar to (16), and corresponding integrals may have to be solved numerically. Computing moments of a function or distribution involves integrals which are inherently stable and error-smoothing. The latter allows this technique to naturally filter noise in the experimental data [268]. In addition, since the aim is not necessarily to characterize the entire $w(M)$, the calculation is efficient.

It should be pointed out that there are other methods that cannot be easily classified into one of the three categories above. For example, Wolpert and Ickstadt employed a nonparametric Bayesian inference scheme using Levy random fields as priors and were able to accurately reflect the uncertainty inherent in the inversion process [269]. Similarly, Guzmán et al. [270] described the molecular weight distribution analytically (using GEX functions) assuming $\beta=2$ (double reptation) and avoided the explicit numerical inversion of (2). Other attractive features of their method included an estimate of the uncertainty and a relaxation of the need to specify some material parameters required for other methods.

4.1.1. Frequency Window. The principle of time-temperature superposition allows us to perform rheological measurements at different temperatures and superpose the data to create a single master plot. It is thus possible to measure linear viscoelastic spectra that span 10 decades of frequency or more and observe the high frequency Rouse modes, the plateau region, and the terminal region, where $G^{\prime}(\omega) \sim \omega^{-2}$ and $G^{\prime \prime}(\omega) \sim \omega^{-1}$.

When sufficient experimental data is available in the terminal regime, the reconstruction of the molecular weight distribution is greatly assisted. While the general maxim "more is good" holds, the amount of low-frequency data required for accurate inversion seems to vary between one to three orders of magnitude below the cross-over frequency depending on the polydispersity and chemistry of the sample [231, 261].

A different set of issues are present on the high-frequency end of the spectrum. Due to the so-called Bueche-Flory theorem [271], the dynamic mechanical response of chemically identical polymers in this regime is independent of the molecular weight or architecture. The "double reptation" equation (2) does not contain the contribution of Rouse modes, as the lower limit of the integral is $M_{e}$ and not zero. Thus, the Rouse contribution has to be either (i) subtracted from the experimental data as done heuristically, for example, by Thimm et al. and Léonardi et al. [230, 272, 273], or (ii) added to the $G(t)$ from the double reptation model (2) obviating the need for processing experimental data as done by van Ruymbeke et al. [231]. Indeed, the latter course of action may be more attractive since van Ruymbeke et al. found that including the intermediate regime of frequencies 
where the Rouse modes overlap with entanglement modes provides valuable information.

4.1.2. Calibration. How do we know whether the $w(M)$ inferred from rheology is acceptable? In particular, where do we stand if the $w(M)$ obtained from SEC and rheology are different from each other. Practically, this situation is commonly encountered and knowing how to deal with it is important in scoring different candidates (algorithms) for analytical rheology of LPs and in establishing how much to trust a certain inferred $w(M)$. Unfortunately, the answer to this question is not particularly clear. Consider the case of samples with small amounts of high molecular weight fractions. These fractions may pass undetected by SEC, while their rheological signals may be reflected quite clearly in the inferred $w(M)$. Thus, we expect differences between the two molecular weight distributions when the sample contains high molecular weight tails. Otherwise, if the two molecular weight distributions lie within the confidence interval of the SEC, which is about $2-5 \%$ standard relative error on the weight-averaged molecular weight [231], then they should be considered indistinguishable. Better still, it is possible to perform uncertainty analysis on the $w(M)$ obtained from inverse rheology to judge whether the predictions are statistically different.

A more subtle but related question is "how independent of SEC is the $w(M)$ inferred from rheology?" It may be recalled that the formulation of the inverse problem using double reptation via (2) is not parameter free. Typically these parameters are regressed by fitting the forward problem to experimental linear viscoelasticity. Unfortunately, the $w(M)$ for this calibration sample has to be known a priori and is usually obtained by SEC. Thus, the calibration of the parameters of the inverse rheology problem is tainted by SEC (or any alternative technique used to measure $w(M)$ ). While some of these effects may be mitigated by using a highly monodisperse sample, as van Ruymbeke et al. put it [231], "SEC and rheology are best viewed as complementary methods, with the most information to be gained from critical comparisons and consistency checks between the two."

4.2. Branched Polymers. The use of rheology as an indirect probe of LCB has a rich tradition. Teasing apart the influence of the molecular weight and molecular weight distribution from branching is highly nontrivial, since they often have similar manifestations $[274,275]$. There is a fair amount of diversity in the measurement and analysis of rheology, and the resulting level of branching information provided. Some purely empirical tests measure a single average property such as $\eta_{0}$ or $J_{e}^{0}$ and tell us whether LCB is present or not $[42,276,277]$. On the other end of the spectrum, many model-driven approaches take into account the entire $G^{*}(\omega)$, and other available information, to quantitatively figure out the amount of LCB present.

4.2.1. Experimental Methods. We first consider some purely experimental techniques. It should be pointed out that more successful recent techniques combine measurements from multiple detectors such as SEC viscometry, light scattering and melt rheology [66, 278-282], as discussed later.

Linear Viscoelastic Probes. The use of simple indices, which determine the enhancement of the viscosity due to LCB above the $\eta_{0} \sim M^{3.4}$ curve, for LPs with the same chemistry, is a popular way of quantifying branching. These techniques include the viscosity index of long-chain branching (v.b.i) [283], Dow rheology index (DRI) [284], and long-chain branching index (LCBI) [278]. Sometimes, the intrinsic viscosity $[\eta]$ is used as a proxy for the average molecular weight $[91,278]$, since $[\eta]$, unlike $\eta_{0}$, is relatively insensitive to small amounts of branching. For example, LCBI is used to quantify branching in polyethylenes and is defined as

$$
\mathrm{LCBI}=\frac{\eta_{0}^{0.179}}{4.8[\eta]}-1,
$$

where $\eta_{0}$ is measured in pascals at $190^{\circ} \mathrm{C}$ and $[\eta]$ is measured in $\mathrm{dL} / \mathrm{g}$ at $135^{\circ} \mathrm{C}$ in trichlorobenzene. Unlike DRI, LCBI is relatively independent of molecular weight and molecular weight distribution. By construction, LCBI is equal to zero for pure LPs. Michel introduced an empirically motivated index $g_{\text {rheo }}$ specifically designed for branched isotactic polypropylene by statistically analyzing a large number of experimental samples [285]. Similarly, Tsenogolou and Gotsis [286] and Tian et al. [49] developed semiempirical techniques to quantify LCB in polypropylenes modified by different melt modifying agents.

In similar vein, the steady-state recoverable elastic compliance, $J_{e}^{0}$ is also correlated with LCB; unfortunately, it is also correlated in the same manner with molecular weight distribution which makes it hard to tease the apart the two effects [287-290]. In addition, the measurement of $J_{e}^{0}$ is more time-consuming than that of $\eta_{0}$, and in many practical cases steady state may not even be experimentally attainable [277, 291].

Besides steady-state properties like $\eta_{0}$ and $J_{e}^{0}$, which rely on a single number, numerous studies consider the characteristics of different viscometric functions. Thus, instead of comparing just the $\eta_{0}$ or $J_{e}^{0}$ of linear and branched counterparts, they consider the difference in magnitude and form of entire functions. A popular form involves plotting the modulus of the complex viscosity $\left|\eta^{*}(\omega)\right|$ against the frequency $\omega$ [292-294]. This is related to the Vinogradov plot which plots $\left|\eta^{*}(\omega)\right| / \eta_{0} \mathrm{v} / \mathrm{s} \eta_{0} \omega$ [295]. The presence of LCB is characterized by "wiggles" or inflection points in the $\left|\eta^{*}(\omega)\right|$ v/s $\omega$ plot, which may be discerned more clearly by plotting the slope.

Cole-Cole plot of $G^{\prime \prime}(\omega)$ v/s $G^{\prime}(\omega)$, and van GurpPalmen plot of loss angle $\delta=\tan ^{-1}\left[G^{\prime \prime}(\omega) / G^{\prime}(\omega)\right] \mathrm{v} / \mathrm{s}$ the complex modulus $\left|G^{*}(\omega)\right|$ are other alternatives that are very sensitive to changes in relaxation processes $[88,296-301]$. For example, Robertson et al. [302] used the van GurpPalmen plot and correlated the peak in $\delta$ at intermediate frequencies with LCB by appealing to blending rules. Trinkle et al. [297, 300] found that using a reduced van GurpPalmen plot by plotting $\delta$ against $\left|G^{*}(\omega)\right| / G_{N}^{0}$ allows one to study the effects of molecular weight and molecular weight 
distribution in LPs systematically. The presence of multiple "bumps" on these plots indicates additional relaxation modes in the form of bimodal molecular weight distribution, or, when that can be ruled out, the presence of LCB. Recent studies seem to indicate the superiority of $\delta-\left|G^{*}(\omega)\right|$ over $G^{\prime \prime}(\omega)-G^{\prime}(\omega)$ for LCB detection [303].

Thermorheological Probes. In the linear viscoelastic-based methods above, measurements are effectively carried out at a constant temperature (ignoring, for the moment, the use of time-temperature superposition to create master plots). We can additionally exploit the fact that the rheology of LPs and BPs has distinct thermal signatures. For example, the flow activation energy $E_{a}$ can be measured by considering the temperature dependence of dynamic viscosity. It is found to be a sensitive probe of branching [32, 291, 303]. Unfortunately, the effect does not distinguish between SCB and LCB $[32,304]$. When this disadvantage is combined with the modest magnitude of change in the activation energy from LCB and thermorheological complexity that is common in many branched polyethylenes, it makes for a poor probe [32, 291, 303-306]. However, some researchers have been able to use thermorheological complexity, or the breakdown of time-temperature superposition, to indicate the presence of LCB [291].

Nonlinear Rheology. The bulk of literature on analytical rheology relies on linear viscoelasticity, because experiments are more convenient to perform and less prone to experimental error like wall-slip and shear-banding. However, quite a few studies have considered the difference in the nonlinear shear or extensional response of LPs and BPs [91, 307-314] as a probe of LCB.

In extensional rheology, BPs show the onset of strain hardening at deformation rates far below the inverse Rouse time. This can be used to detect the presence of LCB. It should be pointed out that molecules with no "innersegments" (segments capped on both ends by branch-points) like stars do not show this effect $[44,104,313]$. Thus, it may not be particularly suitable for sparsely branched $\mathrm{mPE}$, for example, which mostly contains linears and stars.

The damping function $h(\gamma)$ in simple shear offers the possibility of being a good probe for LCB, since it is relatively insensitive to molecular weight distribution typically seen in industry [310]. Stadler and Münstedt $[88,315]$ sought to use size-exclusion chromatography with multiangle laser light scattering (SEC-MALLS), and shear rheological measurements to analyze viscosity functions of LCB polyethylenes, by separating the influence of long-chain branching and molecular weight on rheology. Doerpinghaus and Baird [316] parameterized the pom-pom model, which describes the nonlinear rheology of BPs semiquantitatively [53, 163, $165,166,317-320]$, to shear and extensional rheology for different levels of LCB.

A potentially systematic technique for studying the nonlinear response is Fourier transform rheology, or FTrheology [314, 321-326]. This method is similar to the typical linear viscoelastic frequency sweep, except that, instead of small-amplitude oscillatory shear, a large-amplitude oscillatory shear (LAOS) deformation at a particular $\omega, \gamma(t)=$ $\gamma_{0} \sin (\omega t)$ with large $\gamma_{0}$, is applied. This results in the breakdown of Boltzmann superposition principle, and the resulting torque or stress is not a simple harmonic function, and instead contains higher harmonics,

$$
T(t)=I_{1} e^{i \omega t}+I_{3} e^{i 3 \omega t}+I_{5} e^{i 5 \omega t}+\cdots .
$$

Indeed, by performing Fourier analysis on the stress response, higher odd harmonics, such as $I_{3}$ and $I_{5}$, are found to be nonzero.

An analysis of these harmonics, via the ratios $I_{3} / I_{1}$ or $I_{5} / I_{1}$, is found to be a sensitive nonlinear probe of molecular weight distribution and branching [314]. In fact, Vittorias et al. [327] employed a combination of FT rheology [314] and the pom-pom model $[53,166]$ to determine the topology of BPs.

Multidetector Methods. The rheological signature of polymers with broad molecular weight distribution often mimics that of LCB. For example, $J_{e}^{0}$, which is more sensitive to large molecular weight than $\eta_{0}$, can be amplified to a greater degree by high molecular weight components, than by LCB. Similarly, strain hardening at small strain rates due to high molecular weight components can completely mask the contribution of LCB [328]. Thus, an independent measurement of the molecular weight distribution, using SEC or SECMALLS, can help to separate the two effects. Thus, it has now become quite common to complement rheology, with a suite of independent tests to probe LCB.

Janzen and Colby [66] used the phenomenological model developed by Lusignan et al. $[329,330]$ which related $\eta_{0}$ to the average molecular weight and the spacing between branches, in addition to three other chemistry-specific parameters. They were careful to apply an explicit correction to the molecular weight inferred from SEC due to branchinginduced changes to the hydrodynamic volume. Given SEC data and $\eta_{0}$, they could then invert the phenomenological model to estimate the spacing between long-chain branches.

Wood-Adams and Dealy [42] used triple-detector SEC, which allowed them to measure the molecular weight and intrinsic viscosity of fractions emerging from the SEC column. They combined these measurements with linear viscoelasticity. They used an empirical expression, which was later linked to molecular theory [331], to compute the difference between molecular weight distribution curves obtained via chromatography and those inferred from complex viscosity [332], and linked the difference to LCB frequency.

van Ruymbeke et al. [63] used the molecular weight distribution measured by SEC with their time-dependent diffusion reptation model [213] for LPs to obtain a predicted linear viscoelastic response. They developed a criterion to quantify the level of LCB by analyzing the difference between the predicted and measured viscoelastic response. They were able to detect LCB in the 1/1000 backbone C-atoms range which is below the threshold of NMR for high-density polyethylenes synthesized by Zeigler Natta, Phillips, and metallocene catalysts. 
Crosby et al. [333] suggested a dilution rheology method, where the concentration-dependent variation of $\eta_{0}$, in conjunction with molecular theory and rudimentary molecular weight information, was able to quantify the LCB in different families of branched polyethylenes. Krause et al. [334] used electron beam irradiation to introduce branching in isotactic polypropylene and studied it using SEC and linear/nonlinear rheological experiments. They found rheology to be more sensitive to branching, as expected, but reported that a combination of both the methods resulted in better overall characterization. Some of the works previously referenced also employ multidetector methods [88, 219, 220, 302, 315].

In an excellent recent review on diagnosing low levels of branching in metallocene-catalyzed polyethylenes, Stadler [335] evaluated the effectiveness of many different rheological probes at low $(30 \mathrm{~K}-50 \mathrm{~K})$, medium $(50 \mathrm{~K}-120 \mathrm{~K})$, high $(120 \mathrm{~K}-250 \mathrm{~K})$, and ultrahigh $(\geq 250 \mathrm{~K})$ molecular weights. He concluded by advocating multidetector techniques by noting "as small amounts of long-chain branches can have a very similar effect as a high-molecular tail, it is not advisable to use rheological means alone to investigate long-chain branching. It should always be confirmed by SEC (or better by SEC-MALLS) that no such high molecular tails are present." Likewise, in attempting to characterize branched polyacrylates, Castignolles et al. [336] opined that the application of ${ }^{13} \mathrm{C}$ NMR is more complex and suggested combining spectroscopic, chromatographic, and rheological information to fully characterize molecular information.

4.2.2. Model-Driven Data-Analysis Methods. The trend from measuring a single property to diagnose LCB towards processing of multiple bits of information in multidetector techniques is further extended by model-driven methods. Some of the more sophisticated model-driven techniques allow us to combine heterogeneous data like rheology and molecular weight distribution obtained from multiple detectors with molecular models by exploiting some a priori information about the sample. I have already mentioned several methods which explicitly employ models (whether molecular-based or phenomenological) to interpret experimental data $[63,66,316,327,331,333]$. The rest implicitly use a model, even though it may only be specified as an empirical correlation, and not really labeled as a "model." Most studies using molecular-based models use predictions of the linear viscoelasticity of LPs to analyze the difference between the predicted and observed rheology. In the case of studies based on nonlinear rheology, predictions of the pompom model are used in some approximate manner, since it should be reiterated that the pom-pom model is not a general model for the prediction of the nonlinear rheological response of arbitrary mixtures of BPs. Despite the availability of hierarchical models for the prediction of the linear viscoelasticity of mixtures of BPs, they have seen limited use in analytical rheology.

Perhaps, the principal reason for this is the inability to construct an approximation to the full hierarchical model. That is, there is no counterpart to the idea of double reptation, which allows us to approximately capture the linear viscoelastic behavior of BPs and state the inverse problem as a single equation like (2). However, in the 20 years since the first systematic applications of analytical rheology for LPs, computers have become significantly powerful, and perhaps the need for an approximate model is overstated. Even if we neglect computational cost, there are methodological challenges that have to be surmounted to cast the inverse problem in a systematic form. Thus, in the following, we will focus primarily on recently introduced techniques for the inversion of the full hierarchical models for BPs.

As mentioned earlier, model-driven methods work best when additional information on the unknown sample is available. Information about the sample may be available in the form of a good understanding of the underlying polymerization kinetics, or through knowledge of blending processes (if two separately synthesized samples have been mixed). For example, the polymerization mechanism of single-site metallocene-polyethylenes, which are commercially important, is relatively well understood. It can be characterized surprisingly well by only two parameters, the average molecular weight and the level of branching, even though the actual sample contains a mixture linear, star, $\mathrm{H}$-shaped, comb, and hyperbranched molecules [337, 338]. Algorithms for the generation of molecules of different shapes and sizes for various kinds of polymerizations have been developed by Tobita and coworkers [339-344], which can be helpful to reduce the "dimensionality" of the inverse problem by allowing the description of a complex ensemble of molecules using only a few parameters.

If the sample has been prepared by blending two samples of different types, it is useful to know the blending ratio in addition to the polymerization mechanisms by which the two initial samples were created, so that the ensemble of molecules can potentially be constructed [345]. Once we are able to describe the molecules parametrically (using a few parameters), we can apply molecular models based on the tube theory.

Inversion of Hierarchical Models. Recently, a Bayesian formulation of the problem of analytical rheology of BP was reported [346-348], which imposed a systematic modeldriven computational framework, without sacrificing the flexibility of integrating heterogeneous pieces of information. Compared to the simple indices mentioned earlier, this technique is mathematically more complex, computationally far more demanding, and, because of its probabilistic outlook, sometimes harder to interpret.

The central insight of this technique is a movement away from the most common method of posing inverse problems in rheology, namely, by asking the question [347] "what structure and composition minimizes the distance (often measured in a least-squared sense) between experimental data and model predictions?" This conventional approach reduces analytical rheology into an optimization problem, as employed in the analytical rheology of LPs using parameterization or regularization. Of all the issues afflicting inverse problems in general $[349,350]$, the most serious for the analytical rheology of BPs in particular is the treatment of 
multiple solutions, or the prevalence of different mixtures which give rise to approximately the same rheology. Problems of "degeneracy" are significantly compounded for BPs when compared with LPs, not only due to the richness of potential mixtures and linear viscoelastic responses, but also due to the additional uncertainty introduced by more sophisticated models and experiments.

Different techniques adopt different strategies for dealing with degeneracy; multidetector methods discriminate between potential solutions by using multiple signals to weed out some structures which may be compatible with the rheology, but incompatible with SEC data, for example. In other techniques, parsimony is imposed from above to protect against overfitting the data: Thus, in the analytical rheology of LPs, parameterization techniques restrict the available degrees of freedom by only admitting solutions that conform with the prescribed parameterization, while regularization methods add a smoothness requirement to fence out the degenerate solutions.

This makes interpretation of the results of optimizationbased techniques straightforward, since we get a "best" solution defined in some sense. However, they suffer from several drawbacks, due to their implicit assumption of uniqueness. They are not equipped to address or describe the existence of multiple solutions meaningfully. The Bayesian formulation transforms the inverse problem into a sampling problem, which can then be investigated using a Markov-chain Monte Carlo algorithm. By design, it avoids the trap that optimization-based methods suffer from and explores the distribution of structures and compositions consistent with a certain linear viscoelastic response. When properly implemented, it is capable of describing all possible solutions and assigning them with a degree of likelihood. When applied to mixtures of linears and stars, for example, it was shown [346] that the method was able to (i) identify the number of components in an unknown mixture (whether single component or blend) due to the intrinsic Occam's razor [351-353], (ii) describe the composition of the mixtures in the absence of degeneracy, and (iii) describe multiple solutions, when it was not possible to rule them out. Further work [347] confronted the last of these issues, by recognizing that, while identification of degeneracy is important, it is only the first step in its ultimate resolution. It provided an algorithm inspired by the Larson's idea [93] of combinatorial rheology or "performing rheological studies of partially characterized samples with fully characterized samples of known molecular weight and branching" to suggest the best possible experiments to perform to discriminate between the multiple solutions. It should be recognized that degeneracy cannot be resolved without performing additional experiments. More recently, this technique has been successfully applied to commercial metallocene-catalyzed polyethylenes [348], using multiple sources of data (rheology and SEC) to infer the probability distribution of the level of branching.

Inversion of Nonlinear Models. As mentioned several times before, the pom-pom is the only BP for which a reasonable nonlinear rheology model exists. Until the development of constitutive equations for more complex branched architectures, the best we can do is to attempt to associate a particular BP with a "similar" pom-pom polymer. Indeed, the idea of combining the results of a recent study by Read et al. [10] with the aforementioned Bayesian framework is very promising. Read et al. considered LDPE, a polymer of immense commercial importance, which is known to be particularly hard to model because of its highly branched nature. Using a 4-parameter polymerization model for the high pressure free radical synthesis, they fit the parameters using SEC (via $g$-ratio analysis) and linear viscoelastic data (using the $\mathrm{BoB}$ model [144]), enormously reducing the dimensionality of the problem. From the ensemble of molecules generated by the polymerization model using the fitted parameters, they mapped the priority (related to the number of free ends connected to an internal segment) and relaxation time distributions to pom-pom modes [163]. The predictions for shear and extensional deformations of the pom-pom model for the mapped structure agreed very well with the experiments. While this study did not directly invert the pom-pom model (using it for validation, instead), it is easy to envision how it could potentially be incorporated into the Bayesian framework.

\section{Summary and Perspective}

The unusual sensitivity of rheology to molecular structure makes it an attractive candidate as a tool for inferring structure from simple rheological measurements, where the sensitivity is translated into resolution of the analytical tool. This typically requires the inversion of a forward model (as shown in Figure 1), which may be a simple correlation or empirical thumb-rule (e.g., indices to infer LCB), a simplified toy version of a more complicated model (e.g., use of simple double-reptation-type models for inferring the molecular weight distribution of LPs), simplified applications of incomplete models (e.g., use of pom-pom model to map the nonlinear rheology of complex BPs), or a full-blown sophisticated model capable of handing arbitrary mixtures of polymers of arbitrary mixtures (e.g., hierarchical models for linear viscoelasticity such as BoB). In addition to resolution, the extreme sensitivity of rheology also offers modest protection from the imperfection of these forward models (Figure 4). Analytical rheology also offers other important advantages over other analytical techniques. It is easier and cheaper to perform, more sensitive to precisely those components that have the greatest relevance to processing, and circumvents the dissolution step, which offers its own set of challenges.

For mixtures of LPs, the empirical validity of the doublereptation picture enables us to formulate the problem of analytical rheology as an inversion of a Fredholm integral of the first kind as shown in (2). The simplified kernel $F(t, M)$ along with the parameter $\beta$, which models constraint release, offers a convenient and empirically reasonable approximation to a more complete and complex theory based on the tube model [142]. The task of solving the inverse problem is usually accomplished by regularization, parameterization, or stabilization. 
For BPs with LCB, the need for analytical rheology is more pressing, since all the other available techniques including $g$-ratio analysis and spectroscopy are seriously flawed in one way or another. The general problem of inferring the structure of an arbitrary mixture of polymers of arbitrary architectures is far more complicated than the analytical rheology of LPs and, in this very general sense, remains unsolved and perhaps potentially unsolvable. That does not necessarily imply that meaningful information cannot be extracted for important subclasses of the general problem. Indeed the analytical rheology of LPs is a subset of the BPs enunciated in this general form. If the chemistry of the polymer is specified (e.g., isotactic polypropylene), there exist methods to diagnose and quantity the extent of LCB, using empirically inspired forward models. There is a general consensus that aggregation of multiple sources of data such as rheology and SEC-MALLS is better than either of the sources alone, although there is no clear consensus on manner in which the aggregation should best be carried out and interpreted. If the polymerization kinetics of the polymer sample are well established, then molecular model-driven methods can be employed to infer the branching information using recent Bayesian data-analysis methods, which currently offer the most sophisticated treatment of degeneracy. In addition to linear rheology, the force of nonlinear rheology may also be brought to bear upon the problem-perhaps through a systematic technique like FT-rheology. While there is currently no general theory capable of modeling the nonlinear rheology of arbitrary BPs, it is an area of intense research. Additionally, mapping complicated BPs to the pom-pom model has been modestly successful and can be used as a stop-gap measure until more progress is made in modeling nonlinear rheology.

In closing, it is useful to emphasize once again what analytical rheology can do well and what it cannot. Rheology is a very sensitive probe of certain features of polymer architecture like molecular weight and LCB. These features are extremely significant for many commercially important synthetic polymers (primarily polyolefins) and that is the niche that analytical rheology occupies. But the potential heterogeneities, even while confining ourselves to synthetic polymers, extend far beyond that. Consider the example of poly(vinyl butyral) [19] which is used as an interlayer in laminated safety glass. It shows polydispersity in molar mass, chemical composition (it is a copolymer), SCB, and LCB. Sometimes it also exhibits crosslinking and graft-induced branching. In addition, the presence of intra- and intermolecular hydrogen bonding compounds dissolution, and, unlike well-studied polymers such as polyethylene, it lacks well-characterized narrow molecular weight linear standards. Clearly, even with perfect models and techniques for direct and inverse rheology, one cannot imagine fully characterizing such a polymer, using rheological techniques alone.

\section{Acknowledgment}

Support from the National Science Foundation under NSF DMR 0953002 is gratefully acknowledged.

\section{References}

[1] R. G. Larson, The Structure and Rheology of Complex Fluids, Oxford University Press, New York, NY, USA, 1998.

[2] F. A. Morrison, Understanding Rheology, Oxford University Press, New York, NY, USA, 2001.

[3] C. W. Macosko, Rheology: Principles, Measurements, and Applications, Wiley-VCH, New York, NY, USA, 1994.

[4] J. M. Franco, M. Berjano, and C. Gallegos, "Linear viscoelasticity of salad dressing emulsions," Journal of Agricultural and Food Chemistry, vol. 45, no. 3, pp. 713-719, 1997.

[5] M. C. Sanchez, M. Berjano, A. Guerrero, E. Brito, and C. Gallegos, "Evolution of the microstructure and rheology of O/W emulsions during the emulsification process," Canadian Journal of Chemical Engineering, vol. 76, no. 3, pp. 479-485, 1998.

[6] T. G. Mason, "New fundamental concepts in emulsion theology," Current Opinion in Colloid and Interface Science, vol. 4, no. 3, pp. 231-238, 1999.

[7] L. R. Hutchings, S. M. Kimani, D. M. Hoyle et al., "In silico molecular design, synthesis, characterization, and rheology of dendritically branched polymers: closing the design loop," ACS Macro Letters, vol. 1, no. 3, pp. 404-408, 2012.

[8] S. Ahmad, B. F. Johnston, S. P. Mackay et al., "In silico modelling of drug-polymer interactions for pharmaceutical formulations," Journal of the Royal Society Interface, vol. 7, supplement 4, pp. S423-S433, 2010.

[9] R. Vaidyanathan and M. El-Halwagi, "Computer-aided design of high performance polymers," Journal of Elastomers and Plastics, vol. 26, no. 3, pp. 277-293, 1994.

[10] D. J. Read, D. Auhl, C. Das et al., "Linking models of polymerization and dynamics to predict branched polymer structure and flow," Science, vol. 333, no. 6051, pp. 18711874, 2011.

[11] N. Adams and P. Murray-Rust, "Engineering polymer informatics: towards the computer-aided design of polymers," Macromolecular Rapid Communications, vol. 29, no. 8, pp. 615-632, 2008.

[12] R. J. Young and P. A. Lovell, Introduction to Polymers, CRC Press, Boca Raton, Fla, USA, 3rd edition, 2011.

[13] A. H. Willbourn, "Polymethylene and the structure of polyethylene: study of short-chain branchingits nature and effects," Journal of Polymer Science A, vol. 34, no. 127, pp. 569-597, 1959.

[14] C. A. Sperati, W. A. Franta, and H. W. Starkweather, "The molecular structure of polyethylene. V. The effect of chain branching and molecular weight on physical properties," Journal of the American Chemical Society, vol. 75, no. 24, pp. 6127-6133, 1953.

[15] F. A. Bovey, F. C. Schilling, F. L. McCrackin, and H. L. Wagner, "Short-chain and long-chain branching in low-density polyethylene," Macromolecules, vol. 9, no. 1, pp. 76-80, 1976.

[16] P. J. Flory, Principles of Polymer Chemistry, Cornell University, Ithaca, NY, USA, 1953.

[17] P. G. de Gennes, "Reptation of stars," Journal de Physique, vol. 36, no. 12, pp. 1199-1203, 1975.

[18] J. M. Dealy and R. G. Larson, Molecular Structure and Rheology of Molten Polymers, Hanser, Munich, Germany, 1st edition, 2006.

[19] A. M. Striegel, "Separation science of macromolecules: What is the role of multidetector size-exclusion chromatography?" in ACS Symposium Series, vol. 893, pp. 2-12, ACS Publications, 2005. 
[20] M. Gaborieau and P. Castignolles, "Size-exclusion chromatography (SEC) of branched polymers and polysaccharides," Analytical and Bioanalytical Chemistry, vol. 399, no. 4, pp. 1413-1423, 2011.

[21] C. Gao and D. Yan, "Hyperbranched polymers: from synthesis to applications," Progress in Polymer Science, vol. 29, no. 3, pp. 183-275, 2004.

[22] E. A. Jordan, A. M. Donald, L. J. Fetters, and J. Klein, "Transition from linear to star-branched diffusion in entangled polymer melts," Polymer Preprints, vol. 30, pp. 63-64, 1989.

[23] C. B. Gell, W. W. Graessley, V. Efstratiadis, M. Pitsikalis, and N. Hadjichristidis, "Viscoelasticity and self-diffusion in melts of entangled asymmetric star polymers," Journal of Polymer Science B, vol. 35, no. 12, pp. 1943-1954, 1997.

[24] K. F. Freed and J. Dudowicz, "Influence of short chain branching on the miscibility of binary polymer blends: application to polyolefin mixtures," Macromolecules, vol. 29, no. 2, pp. 625-636, 1996.

[25] Y. M. Kim and J. K. Park, "Effect of short chain branching on the blown film properties of linear low density polyethylene," Journal of Applied Polymer Science, vol. 61, no. 13, pp. 23152324, 1996.

[26] X. Xu, J. Xu, L. Feng, and W. Chen, "Effect of short chainbranching distribution on crystallinity and modulus of metallocenebased ethylene-butene copolymers," Journal of Applied Polymer Science, vol. 77, no. 8, pp. 1709-1715, 2000.

[27] C. Li Pi Shan, J. B. P. Soares, and A. Penlidis, "Mechanical properties of ethylene/1-hexene copolymers with tailored short chain branching distributions," Polymer, vol. 43, no. 3, pp. 767-773, 2002.

[28] P. Gupta, G. L. Wilkes, A. M. Sukhadia et al., "Does the length of the short chain branch affect the mechanical properties of linear low density polyethylenes? An investigation based on films of copolymers of ethylene/1-butene, ethylene/1-hexene and ethylene/1-octene synthesized by a single site metallocene catalyst," Polymer, vol. 46, no. 20, pp. 8819-8837, 2005.

[29] S. Acierno and P. van Puyvelde, "Effect of short chain branching upon the crystallization of model polyamides-11," Polymer, vol. 46, no. 23, pp. 10331-10338, 2005.

[30] F. J. Stadler, C. Gabriel, and H. Münstedt, "Influence of shortchain branching of polyethylenes on the temperature dependence of rheological properties in shear," Macromolecular Chemistry and Physics, vol. 208, no. 22, pp. 2449-2454, 2007.

[31] R. K. Krishnaswamy, Q. Yang, L. Fernandez-Ballester, and J. A. Kornfield, "Effect of the distribution of short-chain branches on crystallization kinetics and mechanical properties of high-density polyethylene," Macromolecules, vol. 41, no. 5, pp. 1693-1704, 2008.

[32] U. Kessner, J. Kaschta, F. J. Stadler, C. S. Le Duff, X. Drooghaag, and H. Münstedt, "Thermorheological behavior of various short-and long-chain branched polyethylenes and their correlations with the molecular structure," Macromolecules, vol. 43, no. 17, pp. 7341-7350, 2010.

[33] A. D. Channell and E. Q. Clutton, "The effects of short chain branching and molecular weight on the impact fracture toughness of polyethylene," Polymer, vol. 33, no. 19, pp. 4108-4112, 1992.

[34] B. Monrabal, "Crystallization analysis fractionation: a new technique for the analysis of branching distribution in polyolefins," Journal of Applied Polymer Science, vol. 52, no. 4, pp. 491-499, 1994.

[35] J. B. P. Soares, B. Monrabal, J. Nieto, and J. Blanco, "Crystallization analysis fractionation (CRYSTAF) of poly (ethylene-co-1-octene) made with single-site-type catalysts: a mathematical model for the dependence of composition distribution on molecular weight," Macromolecular Chemistry and Physics, vol. 199, no. 9, pp. 1917-1926, 1998.

[36] S. Anantawaraskul, J. B. P. Soares, and P. M. Wood-Adams, "Effect of operation parameters on temperature rising elution fractionation and crystallization analysis fractionation," Journal of Polymer Science B, vol. 41, no. 14, pp. 1762-1778, 2003.

[37] D. M. Sarzotti, J. B. P. Soares, L. C. Simon, and L. J. D. Britto, "Analysis of the chemical composition distribution of ethylene/ $\alpha$ - olefin copolymers by solution differential scanning calorimetry: an alternative technique to Crystaf," Polymer, vol. 45, no. 14, pp. 4787-4799, 2004.

[38] S. Anantawaraskul, J. B. P. Soares, and P. M. Wood-Adams, "Fractionation of semicrystalline polymers by crystallization analysis fractionation and temperature rising elution fractionation," Advances in Polymer Science, vol. 182, pp. 1-54, 2005.

[39] J. B. P. Soares, "An overview of important microstructural distributions for polyolefin analysis," Macromolecular Symposia, vol. 257, pp. 1-12, 2007.

[40] P. M. Wood-Adams, J. M. Dealy, A. W. DeGroot, and O. D. Redwine, "Effect of molecular structure on the linear viscoelastic behavior of polyethylene," Macromolecules, vol. 33, no. 20, pp. 7489-7499, 2000.

[41] G. M. Benedikt and B. L. Goodall, Metallocene-Catalyzed Polymers: Materials, Properties, Processing \& Markets, Plastics Design Library, Norwich, NY, USA, 1998.

[42] P. M. Wood-Adams and J. M. Dealy, "Using rheological data to determine the branching level in metallocene polyethylenes," Macromolecules, vol. 33, no. 20, pp. 7481-7488, 2000.

[43] W. W. Graessley, "Effect of long branches on the flow properties of polymers," Accounts of Chemical Research, vol. 10, no. 9, pp. 332-339, 1977.

[44] D. J. Lohse, S. T. Milner, L. J. Fetters et al., "Well-defined, model long chain branched polyethylene. 2. Melt rheological behavior," Macromolecules, vol. 35, no. 8, pp. 3066-3075, 2002.

[45] J. Vega, M. Aguilar, J. Peon, D. Pastor, and J. Martinez-Salazar, "Effect of long chain branching on linear-viscoelastic melt properties of polyolefins," E-Polymers, vol. 46, pp. 1-35, 2002.

[46] R. S. Porter, J. P. Knox, and J. F. Johnson, "On the flow and activation energy of branched polyethylene melts," Journal of Rheology, vol. 12, no. 3, p. 409, 1968.

[47] X. Wang, C. Tzoganakis, and G. L. Rempel, "Chemical modification of polypropylene with peroxide/pentaerythritol triacrylate by reactive extrusion," Journal of Applied Polymer Science, vol. 61, no. 8, pp. 1395-1404, 1996.

[48] R. P. Lagendijk, A. H. Hogt, A. Buijtenhuijs, and A. D. Gotsis, "Peroxydicarbonate modification of polypropylene and extensional flow properties," Polymer, vol. 42, no. 25, pp. 10035-10043, 2001.

[49] J. Tian, W. Yu, and C. Zhou, "The preparation and rheology characterization of long chain branching polypropylene," Polymer, vol. 47, no. 23, pp. 7962-7969, 2006.

[50] F. Yoshii, K. Makuuchi, S. Kikukawa, T. Tanaka, J. Saitoh, and K. Koyama, "High-melt-strength polypropylene with electron beam irradiation in the presence of polyfunctional monomers," Journal of Applied Polymer Science, vol. 60, no. 4, pp. 617-623, 1996.

[51] D. Auhl, J. Stange, H. Münstedt et al., "Long-chain branched polypropylenes by electron beam irradiation and their rheological properties," Macromolecules, vol. 37, no. 25, pp. 9465-9472, 2004. 
[52] D. Auhl, F. J. Stadler, and H. Mnstedt, "Comparison of molecular structure and rheological properties of electron-beamand gammairradiated polypropylene," Macromolecules, vol. 45, no. 4, pp. 2057-2065, 2012.

[53] T. C. B. McLeish and R. G. Larson, "Molecular constitutive equations for a class of branched polymers: the pom-pom polymer," Journal of Rheology, vol. 42, no. 1, pp. 81-110, 1998.

[54] S. H. Wasserman and W. W. Graessley, "Effects of polydispersity on linear viscoelasticity in entangled polymer melts," Journal of Rheology, vol. 36, no. 4, pp. 543-572, 1992.

[55] S. Wu, "Polymer molecular-weight distribution from dynamic melt viscoelasticity," Polymer Engineering \& Science, vol. 25, no. 2, pp. 122-128, 1985.

[56] S. Wu, "Dynamic rheology and molecular weight distribution of insoluble polymers: tetrafluoroethylene-hexafluoropropylene copolymers," Macromolecules, vol. 18, no. 10, pp. 2023-2030, 1985.

[57] S. Wu, "Characterization of polymer molecular weight distribution by transient viscoelasticity: polytetrafluoroethylenes," Polymer Engineering \& Science, vol. 28, no. 8, pp. 538-543, 1988.

[58] V. Grinshpun and A. Rudin, "Measurement of markhouwink constants by size exclusion chromatography with a low angle laser light scattering detector," Macromolecular Rapid Communications, vol. 6, no. 4, pp. 219-223, 1985.

[59] A. Rudin, V. Grinshpun, and K. F. O'Driscoll, "Long chain branching in polyethylene," Journal of Liquid Chromatography, vol. 7, no. 9, pp. 1809-1821, 1984.

[60] G. Eder, H. Janeschitz-Kriegl, A. Schausberger, W. Stadlbauer, and G. Schindlauer, "Influence of molar mass distribution on the complex moduli of polymer melts," Journal of Rheology, vol. 33, no. 6, pp. 805-820, 1989.

[61] T. Bremner, A. Rudin, and D. G. Cook, "Melt flow index values and molecular weight distributions of commercial thermoplastics," Journal of Applied Polymer Science, vol. 41, no. 7-8, pp. 1617-1627, 1990.

[62] D. W. Mead, "Determination of molecular weight distributions of linear flexible polymers from linear viscoelastic material functions," Journal of Rheology, vol. 38, no. 6, pp. 1797-1827, 1994.

[63] E. van Ruymbeke, V. Stéphenne, D. Daoust, P. Godard, R. Keunings, and C. Bailly, "A sensitive method to detect very low levels of long chain branching from the molar mass distribution and linear viscoelastic response," Journal of Rheology, vol. 49, no. 6, Article ID 007506JOR, pp. 1503-1520, 2005.

[64] T. J. Kealy and P. L. Pauson, "A new type of organo-iron compound," Nature, vol. 168, no. 4285, pp. 1039-1040, 1951.

[65] G. M. Benedikt, Metallocene-Catalyzed Polymers: Materials, Properties, Processing and Markets, William Andrew, Norwich, NY, USA, 1998.

[66] J. Janzen and R. H. Colby, "Diagnosing long-chain branching in polyethylenes," Journal of Molecular Structure, vol. 485486, pp. 569-584, 1999.

[67] B. H. Zimm and W. H. Stockmayer, "Effect of molecular structure on the linear viscoelastic behavior of polyethylene," Journal of Chemical Physics, vol. 17, no. 12, pp. 1301-1314, 1949.

[68] F. W. Billmeyer Jr., "The molecular structure of polyethylene. III. Determination of long chain branching," Journal of the American Chemical Society, vol. 75, no. 24, pp. 6118-6122, 1953.
[69] E. F. Casassa and G. C. Berry, "Angular distribution of intensity of rayleigh scattering from comblike branched molecules," Journal of Polymer Science A, vol. 4, no. 6, pp. 881-897, 1966.

[70] J. Mazur and F. McCrackin, "Configurational properties of star-branched polymers," Macromolecules, vol. 10, no. 2, pp. 326-332, 1977.

[71] F. L. McCrackin and J. Mazur, "Configuration properties of comb-branched polymers," Macromolecules, vol. 14, no. 5, pp. 1214-1220, 1981.

[72] A. Kolinski and A. Sikorski, "Monte carlo study of starbranched polymers on the tetrahedral lattice. I. Conformation of the macromolecule," Journal of Polymer Science A, vol. 20, no. 11, pp. 3147-3154, 1982.

[73] J. F. Douglas, J. Roovers, and K. F. Freed, "Characterization of branching architecture through "universal" ratios of polymer solution properties," Macromolecules, vol. 23, no. 18, pp. 4168-4180, 1990.

[74] J. E. G. Lipson, "Statistical and metric properties of longchain combs," Macromolecules, vol. 26, no. 1, pp. 203-207, 1993.

[75] J. Freire, "Conformational properties of branched polymers: theory and simulations," in Branched Polymers II, J. Roovers, Ed., vol. 143 of Advances in Polymer Science, pp. 35-112, Springer, Berlin, Germany, 1999.

[76] Y. Wang, I. Teraoka, F. Y. Hansen, G. H. Peters, and O. Hassager, "Mean span dimensions of ideal polymer chains containing branches and rings," Macromolecules, vol. 44, no. 2, pp. 403-412, 2011.

[77] S. Pang and A. Rudin, "Size-exclusion chromatographic assessment of long-chain branch frequency in polyethylenes," in Chromatography of Polymers, chapter 18, pp. 254-269, ACS Publications, 1993.

[78] Y. Yu, P. J. Deslauriers, and D. C. Rohlfing, "SEC-MALS method for the determination of long-chain branching and long-chain branching distribution in polyethylene," Polymer, vol. 46, no. 14, pp. 5165-5182, 2005.

[79] A. M. Striegel, "Long-chain branching macromolecules: SEC analysis," in Encyclopedia of Chromatography, J. Cazes, Ed., pp. 1417-1420, Marcell-Decker, New York, NY, USA, 3rd edition, 2010.

[80] M. E. A. Cudby and A. Bunn, "Determination of chain branching in low density polyethylene by ${ }^{13} \mathrm{C}$ nuclear magnetic resonance and infra-red spectroscopy," Polymer, vol. 17, no. 4, pp. 345-347, 1976.

[81] D. E. Axelson, L. Mandelkern, and G. C. Levy, "13C spin relaxation parameters of branched polyethylenes. Ramifications for quantitative analysis," Macromolecules, vol. 10, no. 3, pp. 557-558, 1977.

[82] A. E. Tonelli, "Comments on the elucidation of stereochemical control in $\alpha$-olefin polymerization via ${ }^{13} \mathrm{C}$ NMR spectroscopy of ethylene-propylene copolymers," Macromolecules, vol. 11, no. 4, pp. 634-636, 1978.

[83] J. C. Randall, F. J. Zoepfl, and J. Silverman, "A ${ }^{13}$ C NMR study of radiation-induced long-chain branching in polyethylene," Makromolekulare Chemie, Rapid Communications, vol. 4, no. 3, pp. 149-157, 1983.

[84] J. C. Randall, "A review of high resolution liquid 13 carbon nuclear magnetic resonance characterizations of ethylenebased polymers," Polymer Reviews, vol. 29, no. 2, pp. 201317, 1989.

[85] J. C. ] Randall, C. J. Ruff, and M. Kelchtermans, " ${ }^{13}$ C NMR microstructure determinations of low-density polyethylene 
homopolymers and copolymers," Recueil des Travaux Chimiques des Pays-Bas, vol. 110, no. 12, pp. 543-552, 1991.

[86] W. Liu, D. G. Ray III, and P. L. Rinaldi, "Resolution of signals from long-chain branching in polyethylene by ${ }^{13} \mathrm{C} \mathrm{NMR}$ at 188.6 MHz," Macromolecules, vol. 32, no. 11, pp. 3817-3819, 1999.

[87] E. W. Hansen, R. Blom, and O. M. Bade, "N.m.r. characterization of polyethylene with emphasis on internal consistency of peak intensities and estimation of uncertainties in derived branch distribution numbers," Polymer, vol. 38, no. 17, pp. 4295-4304, 1997.

[88] F. J. Stadler, C. Piel, W. Kaminsky, and H. Münstedt, "Rheological characterization of long-chain branched polyethylenes and comparison with classical analytical methods," Macromolecular Symposia, vol. 236, pp. 209-218, 2006.

[89] P. Tackx and J. C. J. F. Tacx, "Chain architecture of LDPE as a function of molar mass using size exclusion chromatography and multi-angle laser light scattering (SEC-MALLS)," Polymer, vol. 39, no. 14, pp. 3109-3113, 1998.

[90] K. Klimke, M. Parkinson, C. Piel, W. Kaminsky, H. W. Spiess, and M. Wilhelm, "Optimisation and application of polyolefin branch quantification by melt-state ${ }^{13} \mathrm{C}$ NMR spectroscopy," Macromolecular Chemistry and Physics, vol. 207, no. 4, pp. 382-395, 2006.

[91] A. Malmberg, C. Gabriel, T. Steffl, H. Münstedt, and B. Löfgren, "Long-chain branching in metallocene-catalyzed polyethylenes investigated by low oscillatory shear and uniaxial extensional rheometry," Macromolecules, vol. 35, no. 3, pp. 1038-1048, 2002.

[92] R. N. Shroff and H. Mavridis, "Assessment of NMR and rheology for the characterization of LCB in essentially linear polyethylenes," Macromolecules, vol. 34, no. 21, pp. 73627367, 2001.

[93] R. G. Larson, "Combinatorial rheology of branched polymer melts," Macromolecules, vol. 34, no. 13, pp. 4556-4571, 2001.

[94] F. T. Wall, "Statistical thermodynamics of rubber," Journal of Chemical Physics, vol. 10, pp. 258-271, 1942.

[95] P. J. Flory and J. Rehner, "Statistical mechanics of cross-linked polymer networks I. Rubberlike elasticity," The Journal of Chemical Physics, vol. 11, no. 11, pp. 512-520, 1943.

[96] H. M. James and E. Guth, "Theory of the elastic properties of rubber," Journal of Chemical Physics, vol. 11, no. 10, pp. 455-481, 1943.

[97] L. R. G. Treloar, "The elasticity of a network of long chains," Transactions of the Faraday Society, vol. 39, pp. 241-246, 1943.

[98] P. G. de Gennes, Scaling Concepts in Polymer Physics, Cornell University Press, Ithaca, NY, USA, 1st edition, 1979.

[99] M. Rubinstein and R. H. Colby, Polymer Physics, Oxford University Press, New York, NY, USA, 1st edition, 2003.

[100] R. I. Tanner, Engineering Rheology, vol. 52, Oxford University Press, New York,NY, USA, 2000.

[101] S. F. Edwards and T. A. Vilgis, "The tube model theory of rubber elasticity," Reports on Progress in Physics, vol. 51, no. 2, pp. 243-297, 1988.

[102] M. S. Green and A. V. Tobolsky, "A new approach to the theory of relaxing polymeric media," Journal of Chemical Physics, vol. 14, no. 80, pp. 80-92, 1946.

[103] F. W. Wiegel, "A network model for viscoelastic fluids," Physica, vol. 42, no. 1, pp. 156-164, 1969.

[104] M. Doi and S. F. Edwards, The Theory of Polymer Dynamics, Clarendon Press, Oxford, UK, 1986.

[105] A. S. Lodge, "A network theory of flow birefringence and stress in concentrated polymer solutions," Transactions of the Faraday Society, vol. 52, pp. 120-130, 1956.
[106] M. J. Yamamoto, "The visco-elastic properties of network structure I. general formalism," Journal of the Physical Society of Japan, vol. 11, no. 4, pp. 413-421, 1956.

[107] M. Yamamoto, "The visco-elastic properties of network structure II. structural viscosity," Journal of the Physical Society of Japan, vol. 12, no. 10, pp. 1148-1158, 1957.

[108] M. Yamamoto, "The visco-elastic properties of network structure III. normal stress effect (Weissenberg effect)," Journal of the Physical Society of Japan, vol. 13, no. 10, pp. 1200$1211,1958$.

[109] A. S. Lodge, "A network theory of constrained elastic recovery in concentrated polymer solutions," Rheologica Acta, vol. 1, no. 2-3, pp. 158-163, 1958.

[110] A. S. Lodge, "Constitutive equations from molecular network theories for polymer solutions," Rheologica Acta, vol. 7, no. 4, pp. 379-392, 1968.

[111] W. Kuhn, "Beziehungen zwischen Molekülgröße, statistischer Molekülgestalt und elastischen Eigenschaften hochpolymerer Stoffe," Kolloid-Zeitschrift, vol. 76, no. 3, pp. 258-271, 1936.

[112] H. A. Kramers, "The viscosity of macromolecules in a streaming fluid," Physica, vol. 11, no. 1, pp. 1-9, 1944.

[113] P. E. Rouse, "A theory of the linear viscoelastic properties of dilute solutions of coiling polymers," The Journal of Chemical Physics, vol. 21, no. 7, pp. 1272-1280, 1953.

[114] B. H. Zimm, "Dynamics of polymer molecules in dilute solution: viscoelasticity, flow birefringence and dielectric loss," The Journal of Chemical Physics, vol. 24, no. 2, pp. 269278, 1956.

[115] H. Yamakawa, Modern Theory of Polymer Solutions, Harper \& Row, New York, NY, USA, 1971.

[116] R. Zwanzig, "Theoretical basis for the rouse-zimm model in polymer solution dynamics," The Journal of Chemical Physics, vol. 60, no. 7, pp. 2717-2720, 1974.

[117] M. Bixon, "Polymer dynamics in solution," Annual Review of Physical Chemistry, vol. 27, no. 1, pp. 65-84, 1976.

[118] W. Paul, G. D. Smith, D. Y. Yoon et al., "Chain motion in an unentangled polyethylene melt: A critical test of the rouse model by molecular dynamics simulations and neutron spin echo spectroscopy," Physical Review Letters, vol. 80, no. 11, pp. 2346-2349, 1998.

[119] L. Harnau, R. G. Winkler, and P. Reineker, "Comment on "chain motion in an unentangled polyethylene melt: a critical test of the rouse model by molecular dynamics simulations and neutron spin echo spectroscopy"', Physical Review Letters, vol. 82, no. 11, p. 2408, 2408.

[120] B. Friedman and B. O'Shaughnessy, "Kinetics of intermolecular reactions in dilute polymer solutions and unentangled melts," Macromolecules, vol. 26, no. 21, pp. 5726-5739, 1993.

[121] M. Vladkov and J. L. Barrat, "Linear and nonlinear viscoelasticity of a model unentangled polymer melt: molecular dynamics and rouse modes analysis," Macromolecular Theory and Simulations, vol. 15, no. 3, pp. 252-262, 2006.

[122] V. A. Harmandaris, V. G. Mavrantzas, D. N. Theodorou et al., "Crossover from the rouse to the entangled polymer melt regime: signals from long, detailed atomistic molecular dynamics simulations, supported by rheological experiments,” Macromolecules, vol. 36, no. 4, pp. 1376-1387, 2003.

[123] Q. Sun and R. Faller, "Crossover from unentangled to entangled dynamics in a systematically coarse-grained polystyrene melt," Macromolecules, vol. 39, no. 2, pp. 812-820, 2006.

[124] X. Qiu and M. D. Ediger, "Local and global dynamics of unentangled polyethylene melts by ${ }^{13} \mathrm{C}$ NMR," Macromolecules, vol. 33, no. 2, pp. 490-498, 2000. 
[125] D. R. Hansen, M. C. Williams, and M. Shen, "Viscoelastic properties of entangled polymers. I. The elastically coupled entanglement model," Macromolecules, vol. 9, no. 2, pp. 345354, 1976.

[126] S. D. Hong, D. R. Hansen, M. C. Williams, and M. Shen, "Viscoelastic properties of entangled polymers .2. interchainintrachain entanglement model," Journal of Polymer Science $B$, vol. 15, no. 11, pp. 1869-1883, 1977.

[127] P. G. de Gennes, "Reptation of a polymer chain in the presence of fixed obstacles," The Journal of Chemical Physics, vol. 55, no. 2, pp. 572-579, 1971.

[128] M. Doi and S. F. Edwards, "Dynamics of concentrated polymer systems. Part 1.-Brownian motion in the equilibrium state," Journal of the Chemical Society, Faraday Transactions 2, vol. 74, pp. 1789-1801, 1978.

[129] M. Doi and S. F. Edwards, "Dynamics of concentrated polymer systems. Part 2.-Molecular motion under flow," Journal of the Chemical Society, Faraday Transactions 2, vol. 74, pp. 1802-1817, 1978.

[130] M. Doi and S. F. Edwards, "Dynamics of concentrated polymer systems. Part 3.-The constitutive equation," Journal of the Chemical Society, Faraday Transactions 2, vol. 74, pp. 1818-1832, 1978.

[131] M. Doi and S. F. Edwards, "Dynamics of concentrated polymer systems. Part 4.--Rheological properties," Journal of the Chemical Society, Faraday Transactions 2, vol. 75, pp. 3854, 1979.

[132] D. S. Pearson and E. Helfand, "Viscoelastic properties of starshaped polymers," Macromolecules, vol. 17, no. 4, pp. 888895, 1984.

[133] W. H. Tuminello, "Molecular-weight and molecular-weight distribution from dynamic measurements of polymer melts," Polymer Engineering \& Science, vol. 26, no. 19, pp. 1339-1347, 1986.

[134] J. des Cloizeaux, "Relaxation and viscosity anomaly of melts made of long entangled polymers. Time-dependent reptation," Macromolecules, vol. 23, no. 21, pp. 4678-4687, 1990.

[135] C. Tsenoglou, "Molecular weight polydispersity effects on the viscoelasticity of entangled linear polymers," Macromolecules, vol. 24 , no. 8, pp. 1762-1767, 1991.

[136] R. C. Ball and T. C. B. McLeish, "Dynamic dilution and the viscosity of star polymer melts," Macromolecules, vol. 22, no. 5, pp. 1911-1913, 1989.

[137] M. Doi and N. Y. Kuzuu, "Rheology of star polymers in concentratedsolutions and melts," Journal of Polymer Science C, vol. 18, pp. 775-780, 1980.

[138] W. W. Graessley, "Entangled linear, branched and network polymer systems-molecular theories," Advances in Polymer Science, vol. 47, pp. 67-117, 1982.

[139] J. P. Montfort, G. Marin, and P. Monge, "Effect of tube renewal on the viscoelastic properties of concentrated solutions of polymers," Macromolecules, vol. 19, no. 2, pp. 393399, 1986.

[140] J. L. Viovy, M. Rubinstein, and R. H. Colby, "Constraint release in polymer melts. Tube reorganization versus tube dilation," Macromolecules, vol. 24, no. 12, pp. 3587-3596, 1991.

[141] S. T. Milner and T. C. B. McLeish, "Parameter-free theory for stress relaxation in star polymer melts," Macromolecules, vol. 30, no. 7, pp. 2159-2166, 1997.

[142] A. E. Likhtman and T. C. B. McLeish, "Quantitative theory for linear dynamics of linear entangled polymers," Macromolecules, vol. 35, no. 16, pp. 6332-6343, 2002.
[143] S. J. Park, S. Shanbhag, and R. G. Larson, "A hierarchical algorithm for predicting the linear viscoelastic properties of polymer melts with long-chain branching," Rheologica Acta, vol. 44, no. 3, pp. 319-330, 2005.

[144] C. Das, N. J. Inkson, D. J. Read, M. A. Kelmanson, and T. C. B. McLeish, "Computational linear rheology of general branchon-branch polymers," Journal of Rheology, vol. 50, no. 2, pp. 207-234, 2006.

[145] S. T. Milner and T. C. B. McLeish, "Reptation and contourlength fluctuations in melts of linear polymers," Physical Review Letters, vol. 81, no. 3, pp. 725-728, 1998.

[146] S. T. Milner, T. C. B. McLeish, R. N. Young, A. Hakiki, and J. M. Johnson, "Dynamic dilution, constraint-release, and star-linear blends," Macromolecules, vol. 31, no. 26, pp. 9345-9353, 1998.

[147] D. R. Daniels, T. C. B. McLeish, B. J. Crosby, R. N. Young, and C. M. Fernyhough, "Molecular rheology of comb polymer melts. 1. Linear viscoelastic response," Macromolecules, vol. 34, no. 20, pp. 7025-7033, 2001.

[148] D. R. Daniels, T. C. B. McLeish, R. Kant et al., "Linear rheology of diluted linear, star and model long chain brached polymer melts," Rheologica Acta, vol. 40, no. 5, pp. 413-415, 2001.

[149] Z. Wang, X. Chen, and R. G. Larson, "Comparing tube models for predicting the linear rheology of branched polymer melts," Journal of Rheology, vol. 54, no. 2, pp. 223-260, 2010.

[150] H. Watanabe, "Viscoelasticity and dynamics of entangled polymers," Progress in Polymer Science, vol. 24, no. 9, pp. 1253-1403, 1999.

[151] T. C. B. McLeish, "Tube theory of entangled polymer dynamics," Advances in Physics, vol. 51, no. 6, pp. 1379-1527, 2002.

[152] T. C. B. McLeish, "Hierarchical relaxation in tube models of branched polymers," Europhysics Letters, vol. 6, no. 6, pp. 511-516, 1988.

[153] Q. Zhou and R. G. Larson, "Direct molecular dynamics simulation of branch point motion in asymmetric star polymer melts," Macromolecules, vol. 40, no. 9, pp. 3443-3449, 2007.

[154] T. C. B. McLeish, J. Allgaier, D. K. Bick et al., "Dynamics of entangled H-polymers: theory, rheology, and neutronscattering," Macromolecules, vol. 32, no. 20, pp. 6734-6758, 1999.

[155] R. S. Graham, A. E. Likhtman, T. C. B. McLeish, and S. T. Milner, "Microscopic theory of linear, entangled polymer chains under rapid deformation including chain stretch and convective constraint release," Journal of Rheology, vol. 47, no. 5, pp. 1171-1200, 2003.

[156] J. H. Lee, L. J. Fetters, L. A. Archer, and A. F. Halasa, “Tube dynamics in binary polymer blends," Macromolecules, vol. 38, no. 9, pp. 3917-3932, 2005.

[157] E. van Ruymbeke, R. Keunings, and C. Bailly, "Prediction of linear viscoelastic properties for polydisperse mixtures of entangled star and linear polymers: modified tube-based model and comparison with experimental results," Journal of Non-Newtonian Fluid Mechanics, vol. 128, no. 1, pp. 7-22, 2005.

[158] R. G. Larson, Q. Zhou, S. Shanbhag, and S. J. Park, "Advances in modeling of polymer melt rheology," AIChE Journal, vol. 53, no. 3, pp. 542-548, 2007.

[159] S. Shanbhag, S. J. Park, Q. Zhou, and R. G. Larson, "Implications of microscopic simulations of polymer melts for mean-field tube theories," Molecular Physics, vol. 105, no. 2-3, pp. 249-260, 2007. 
[160] G. Marrucci, F. Greco, and G. Ianniruberto, "Rheology of polymer melts and concentrated solutions," Current Opinion in Colloid and Interface Science, vol. 4, no. 4, pp. 283-287, 1999.

[161] G. Marrucci and G. Ianniruberto, "Open problems in tube models for concentrated polymers," Journal of Non-Newtonian Fluid Mechanics, vol. 82, no. 2-3, pp. 275-286, 1999.

[162] R. G. Larson, T. Sridhar, L. G. Leal, G. H. McKinley, A. E. Likhtman, and T. C. B. McLeish, "Definitions of entanglement spacing and time constants in the tube model," Journal of Rheology, vol. 47, no. 3, pp. 809-818, 2003.

[163] R. J. Blackwell, T. C. B. McLeish, and O. G. Harlen, "Molecular drag-strain coupling in branched polymer melts," Journal of Rheology, vol. 44, no. 1, pp. 121-136, 2000.

[164] K. Lee, M. R. Mackley, T. C. B. McLeish, T. M. Nicholson, and O. G. Harlen, "Experimental observation and numerical simulation of transient "stress fangs" within flowing molten polyethylene," Journal of Rheology, vol. 45, no. 6, pp. 12611276, 2001.

[165] W. M. H. Verbeeten, G. W. M. Peters, and F. P. T. Baaijens, "Differential constitutive equations for polymer melts: the extended Pom-Pom model," Journal of Rheology, vol. 45, no. 4, pp. 823-843, 2001.

[166] N. J. Inkson, T. G. B. McLeish, O. G. Harlen, and D. J. Groves, "Predicting low density polyethylene melt rheology in elongational and shear flows with pom-pom constitutive equations," Journal of Rheology, vol. 43, no. 4, pp. 873-896, 1999.

[167] C. C. Hua and J. D. Schieber, "Segment connectivity, chainlength breathing, segmental stretch, and constraint release in reptation models. I. Theory and single-step strain predictions," Journal of Chemical Physics, vol. 109, no. 22, pp. 10018-10027, 1998.

[168] M. Doi and J. Takimoto, "Molecular modelling of entanglement," Philosophical Transactions of the Royal Society of London A, vol. 361, pp. 641-650, 2003.

[169] Y. Masubuchi, J. I. Takimoto, K. Koyama, G. Ianniruberto, G. Marrucci, and F. Greco, "Brownian simulations of a network of reptating primitive chains," Journal of Chemical Physics, vol. 115, no. 9, pp. 4387-4394, 2001.

[170] S. Shanbhag, R. G. Larson, J. Takimoto, and M. Doi, "Deviations from dynamic dilution in the terminal relaxation of star polymers," Physical Review Letters, vol. 87, no. 19, Article ID 195502, 4 pages, 2001.

[171] J. D. Schieber, J. Neergaard, and S. Gupta, "A full-chain, temporary network model with sliplinks, chain-length fluctuations, chain connectivity and chain stretching," Journal of Rheology, vol. 47, no. 1, pp. 213-233, 2003.

[172] Y. Masubuchi, G. Ianniruberto, F. Greco, and G. Marrucci, "Entanglement molecular weight and frequency response of sliplink networks," Journal of Chemical Physics, vol. 119, no. 13, pp. 6925-6930, 2003.

[173] S. Shanbhag and R. G. Larson, "A slip-link model of branchpoint motion in entangled polymers," Macromolecules, vol. 37, no. 21, pp. 8160-8166, 2004.

[174] Y. Masubuchi, G. Ianniruberto, F. Greco, and G. Marrucci, "Molecular simulations of the long-time behaviour of entangled polymeric liquids by the primitive chain network model," Modelling and Simulation in Materials Science and Engineering, vol. 12, no. 3, pp. S91-S100, 2004.

[175] T. Yaoita, T. Isaki, Y. Masubuchi et al., "Highly entangled polymer primitive chain network simulations based on dynamic tube dilation," Journal of Chemical Physics, vol. 121, no. 24 , pp. 12650-12654, 2004.
[176] D. M. Nair and J. D. Schieber, "Linear viscoelastic predictions of a consistently unconstrained brownian slip-link model," Macromolecules, vol. 39, no. 9, pp. 3386-3397, 2006.

[177] A. E. Likhtman, "Single-chain slip-link model of entangled polymers: simultaneous description of neutron spin-echo, rheology, and diffusion," Macromolecules, vol. 38, no. 14, pp. 6128-6139, 2005.

[178] Y. Masubuchi, H. Watanabe, G. Ianniruberto, F. Greco, and G. Marrucci, "Comparison among slip-link simulations of bidisperse linear polymer melts," Macromolecules, vol. 41, no. 21, pp. 8275-8280, 2008.

[179] R. G. Larson, L. E. Scriven, and H. T. Davis, "Monte Carlo simulation of model amphiphile-oil-water systems," The Journal of Chemical Physics, vol. 83, no. 5, pp. 2411-2420, 1985.

[180] I. Carmesin and K. Kremer, "The bond fluctuation method: a new effective algorithm for the dynamics of polymers in all spatial dimensions," Macromolecules, vol. 21, no. 9, pp. 28192823, 1988.

[181] H. P. Deutsch and K. Binder, "Interdiffusion and self-diffusion in polymer mixtures: a Monte Carlo study," The Journal of Chemical Physics, vol. 94, no. 3, pp. 2294-2304, 1991.

[182] J. Scott Shaffer, "Effects of chain topology on polymer dynamics: bulk melts," The Journal of Chemical Physics, vol. 101, no. 5, pp. 4205-4213, 1994.

[183] A. D. Mackie, A. Z. Panagiotopoulos, D. Frenkel, and S. K. Kumar, "Constant-pressure Monte Carlo simulations for lattice models," Europhysics Letters, vol. 27, no. 7, pp. 549554, 1994.

[184] J. Scott Shaffer, "Effects of chain topology on polymer dynamics: configurational relaxation in polymer melts," The Journal of Chemical Physics, vol. 103, no. 2, pp. 761-772, 1995.

[185] J. S. Shaffer, "Dynamics of confined polymer melts: topology and entanglement," Macromolecules, vol. 29, no. 3, pp. 10101013, 1996.

[186] G. Xu, J. Ding, and Y. Yang, "Monte Carlo simulation of selfavoiding lattice chains subject to simple shear flow. I. Model and simulation algorithm," Journal of Chemical Physics, vol. 107, no. 10, pp. 4070-4084, 1997.

[187] A. van Heukelum and G. T. Barkema, "Lattice models of DNA electrophoresis," Electrophoresis, vol. 23, no. 16, pp. 2562-2568, 2002.

[188] S. Shanbhag and R. G. Larson, "Chain retraction potential in a fixed entanglement network," Physical Review Letters, vol. 94, no. 7, Article ID 076001, 2005.

[189] S. Shanbhag and R. G. Larson, "Identification of topological constraints in entangled polymer melts using the bondfluctuation model," Macromolecules, vol. 39, no. 6, pp. 24132417, 2006.

[190] H. Lin, W. L. Mattice, and E. D. von Meerwall, "Chain dynamics of bidisperse polyethylene melts: a Monte Carlo study on a high-coordination lattice," Macromolecules, vol. 40, no. 4, pp. 959-966, 2007.

[191] G. Subramanian and S. Shanbhag, "On the relationship between two popular lattice models for polymer melts," Journal of Chemical Physics, vol. 129, no. 14, Article ID 144904, 2008.

[192] K. Kremer, G. S. Grest, and I. Carmesin, "Crossover from rouse to reptation dynamics: a molecular-dynamics simulation," Physical Review Letters, vol. 61, no. 5, pp. 566-569, 1988. 
[193] K. Kremer and G. S. Grest, "Dynamics of entangled linear polymer melts: a molecular-dynamics simulation," The Journal of Chemical Physics, vol. 92, no. 8, pp. 5057-5086, 1990.

[194] K. Binder, Monte Carlo and Molecular Dynamics Simulations in Polymer Science, Oxford University Press, Oxford, UK, 1986.

[195] S. Plimpton, "Fast parallel algorithms for short-range molecular dynamics," Journal of Computational Physics, vol. 117, no. 1, pp. 1-19, 1995.

[196] M. Kröger and S. Hess, "Rheological evidence for a dynamical crossover in polymer melts via nonequilibrium molecular dynamics," Physical Review Letters, vol. 85, no. 5, pp. 1128$1131,2000$.

[197] R. Auhl, R. Everaers, G. S. Grest, K. Kremer, and S. J. Plimpton, "Equilibration of long chain polymer melts in computer simulations," Journal of Chemical Physics, vol. 119, no. 24, pp. 12718-12728, 2003.

[198] G. D. Smith, D. Bedrov, L. Li, and O. Byutner, "A molecular dynamics simulation study of the viscoelastic properties of polymer nanocomposites," Journal of Chemical Physics, vol. 117, no. 20, pp. 9478-9490, 2002.

[199] M. Kröger, "Simple models for complex nonequilibrium fluids," Physics Reports, vol. 390, no. 6, pp. 453-551, 2004.

[200] S. Sen, S. K. Kumar, and P. Keblinski, "Viscoelastic properties of polymer melts from equilibrium molecular dynamics simulations," Macromolecules, vol. 38, no. 3, pp. 650-653, 2005.

[201] Q. Zhou and R. G. Larson, "Primitive path identification and statistics in molecular dynamics simulations of entangled polymer melts," Macromolecules, vol. 38, no. 13, pp. 57615765, 2005.

[202] Q. Zhou and R. G. Larson, "Direct calculation of the tube potential confining entangled polymers," Macromolecules, vol. 39, no. 19, pp. 6737-6743, 2006.

[203] R. G. Larson, "Looking inside the entanglement "tube" using molecular dynamics simulations," Journal of Polymer Science B, vol. 45, no. 24, pp. 3240-3248, 2007.

[204] A. E. Likhtman, S. K. Sukumaran, and J. Ramirez, "Linear viscoelasticity from molecular dynamics simulation of entangled polymers," Macromolecules, vol. 40, no. 18, pp. 67486757, 2007.

[205] Z. Wang and R. G. Larson, "Constraint release in entangled binary blends of linear polymers: a molecular dynamics study," Macromolecules, vol. 41, no. 13, pp. 4945-4960, 2008.

[206] M. J. Struglinski and W. W. Graessley, "Effects of polydispersity on the linear viscoelastic properties of entangled polymers. 1. Experimental observations for binary mixtures of linear polybutadiene," Macromolecules, vol. 18, no. 12, pp. 2630-2643, 1985.

[207] A. Schausberger, G. Schindlauer, and H. Janeschitz-Kriegl, "Linear elastico-viscous properties of molten standard polystyrenes-I. Presentation of complex moduli; role of short range structural parameters," Rheologica Acta, vol. 24, no. 3, pp. 220-227, 1985.

[208] Y. Matsumiya, H. Watanabe, and K. Osaki, "Comparison of dielectric and viscoelastic relaxation functions of cis-polyisoprenes: test of tube dilation molecular picture," Macromolecules, vol. 33, no. 2, pp. 499-506, 2000.

[209] S. Wang, S. Q. Wang, A. Halasa, and W. L. Hsu, "Relaxation dynamics in mixtures of long and short chains: tube dilation and impeded curvilinear diffusion," Macromolecules, vol. 36, no. 14, pp. 5355-5371, 2003.

[210] S. Wang, E. D. von Meerwall, S. Q. Wang et al., "Diffusion and rheology of binary polymer mixtures," Macromolecules, vol. 37, no. 4, pp. 1641-1651, 2004.
[211] K. Fuchs, C. Friedrich, and J. Weese, "Viscoelastic properties of narrow-distribution poly(methyl methacrylates)," Macromolecules, vol. 29, no. 18, pp. 5893-5901, 1996.

[212] C. Y. Liu, R. Keunings, and C. Bailly, "Do deviations from reptation scaling of entangled polymer melts result from single- or many-crhain effects?" Physical Review Letters, vol. 97, no. 24, Article ID 246001, 2006.

[213] E. van Ruymbeke, R. Keunings, V. Stéphenne, A. Hagenaars, and C. Bailly, "Evaluation of reptation models for predicting the linear viscoelastic properties of entangled linear polymers," Macromolecules, vol. 35, no. 7, pp. 2689-2699, 2002.

[214] J. Cloizeaux, "Double reptation vs. simple reptation in polymer melts," Europhysics Letters, vol. 5, no. 5, pp. 437-442, 1988.

[215] L. R. Hutchings, "Complex branched polymers for StructureProperty correlation studies: the case for temperature gradient interaction chromatography analysis," Macromolecules, vol. 45, no. 14, pp. 5621-5639, 2012.

[216] X. Chen, M. S. Rahman, H. Lee, J. Mays, T. Chang, and R. Larson, "Combined synthesis, TGIC characterization, and rheological measurement and prediction of symmetric $\mathrm{H}$ polybutadienes and their blends with linear and star-shaped polybutadienes," Macromolecules, vol. 44, no. 19, pp. 7799 7809, 2011.

[217] M. S. Rahman, R. Aggarwal, R. G. Larson, J. M. Dealy, and J. Mays, "Synthesis and dilute solution properties of welldefined H-shaped polybutadienes," Macromolecules, vol. 41, no. 21, pp. 8225-8230, 2008.

[218] M. S. Rahman, H. Lee, X. Chen, T. Chang, R. G. Larson, and J. Mays, "Model branched polymers: synthesis and characterization of asymmetric h-shaped polybutadienes," ACS Macro Letters, vol. 1, pp. 537-540, 2012.

[219] S. W. Li, H. E. Park, J. M. Dealy et al., "Detecting structural polydispersity in branched polybutadienes," Macromolecules, vol. 44, no. 2, pp. 208-214, 2011.

[220] S. Ahn, H. Lee, S. Lee, and T. Chang, "Characterization of branched polymers by comprehensive two-dimensional liquid chromatography with triple detection," Macromolecules, vol. 45, no. 8, pp. 3550-3556, 2012.

[221] X. Chen, H. Lee, M. S. Rahman, T. Chang, J. Mays, and R. Larson, "Analytical rheology of asymmetric $\mathrm{H}$-shaped model polybutadiene melts," Macromolecules, vol. 45, no. 14, pp. 5744-5756, 2012.

[222] F. Léonardi, J. C. Majesté, A. Allal, and G. Marin, "Rheological models based on the double reptation mixing rule: the effects of a polydisperse environment," Journal of Rheology, vol. 44, no. 4, pp. 675-692, 2000.

[223] H. Tao, C. I. Huang, and T. P. Lodge, "Correlation length and entanglement spacing in concentrated hydrogenated polybutadiene solutions," Macromolecules, vol. 32, no. 4, pp. 1212-1217, 1999.

[224] S. J. Park and R. G. Larson, "Dilution exponent in the dynamic dilution theory for polymer melts," Journal of Rheo$\log y$, vol. 47, no. 1, pp. 199-211, 2003.

[225] E. van Ruymbeke, Y. Masubuchi, and H. Watanabe, "Effective value of the dynamic dilution exponent in bidisperse linear polymers: from 1 to 4/3," Macromolecules, vol. 45, no. 4, pp. 2085-2098, 2012.

[226] Y. H. Lin, "Number of entanglement strands per cubed tube diameter, a fundamental aspect of topological universality in polymer viscoelasticity," Macromolecules, vol. 20, no. 12, pp. 3080-3083, 1987. 
[227] T. A. Kavassalis and J. Noolandi, "Entanglement scaling in polymer melts and solutions," Macromolecules, vol. 22, no. 6, pp. 2709-2720, 1989.

[228] S. T. Milner, "Predicting the tube diameter in melts and solutions," Macromolecules, vol. 38, no. 11, pp. 4929-4939, 2005.

[229] D. Maier, A. Eckstein, C. Friedrich, and J. Honerkamp, "Evaluation of models combining rheological data with the molecular weight distribution," Journal of Rheology, vol. 42, no. 5, pp. 1153-1173, 1998.

[230] W. Thimm, C. Friedrich, M. Marth, and J. Honerkamp, "On the Rouse spectrum and the determination of the molecular weight distribution from rheological data," Journal of Rheology, vol. 44, no. 2, pp. 429-438, 2000.

[231] E. van Ruymbeke, R. Keunings, and C. Bailly, "Determination of the molecular weight distribution of entangled linear polymers from linear viscoelasticity data," Journal of NonNewtonian Fluid Mechanics, vol. 105, no. 2-3, pp. 153-175, 2002.

[232] J. D. Ferry, Viscoelastic Properties of Polymers, John Wiley \& Sons, Hoboken, NJ, USA, 3rd edition, 1980.

[233] M. Baumgaertel, A. Schausberger, and H. H. Winter, "The relaxation of polymers with linear flexible chains of uniform length," Rheologica Acta, vol. 29, no. 5, pp. 400-408, 1990.

[234] F. R. Schwarzl, "Numerical calculation of storage and loss modulus from stress relaxation data for linear viscoelastic materials," Rheologica Acta, vol. 10, no. 2, pp. 165-173, 1971.

[235] R. S. Anderssen and D. W. Mead, "Theoretical derivation of molecular weight scaling for rheological parameters," Journal of Non-Newtonian Fluid Mechanics, vol. 76, no. 1-3, pp. 299306, 1998.

[236] R. S. Anderssen and M. Westcott, "The molecular weight distribution problem and reptation mixing rules," ANZIAM Journal, vol. 42, no. 1, pp. 26-40, 2000.

[237] J. Honerkamp and J. Weese, "Determination of the relaxation spectrum by a regularization method," Macromolecules, vol. 22, no. 11, pp. 4372-4377, 1989.

[238] N. Orbey and J. M. Dealy, "Determination of the relaxation spectrum from oscillatory shear data," Journal of Rheology, vol. 35, no. 6, pp. 1035-1049, 1991.

[239] M. Baurngaertel, M. E. de Rosa, J. Machado, M. Masse, and H. H. Winter, "The relaxation time spectrum of nearly monodisperse polybutadiene melts," Rheologica Acta, vol. 31, no. 1, pp. 75-82, 1992.

[240] D. W. Mead, "Numerical interconversion of linear viscoelastic material functions," Journal of Rheology, vol. 38, no. 6, pp. 1769-1795, 1994.

[241] H. H. Winter, "Analysis of dynamic mechanical data: Inversion into a relaxation time spectrum and consistency check," Journal of Non-Newtonian Fluid Mechanics, vol. 68, no. 2-3, pp. 225-239, 1997.

[242] C. J. Brabec, H. Rögl, and A. Schausberger, "Investigation of relaxation properties of polymer melts by comparison of relaxation time spectra calculated with different algorithms," Rheologica Acta, vol. 36, no. 6, pp. 667-676, 1997.

[243] R. S. Anderssen and A. R. Davies, "Simple moving-average formulae for the direct recovery of the relaxation spectrum," Journal of Rheology, vol. 45, no. 1, pp. 1-27, 2001.

[244] F. J. Stadler and C. Bailly, "A new method for the calculation of continuous relaxation spectra from dynamic-mechanical data," Rheologica Acta, vol. 48, no. 1, pp. 33-49, 2009.

[245] A. R. Davies and R. S. Anderssen, "Sampling localization in determining the relaxation spectrum," Journal of NonNewtonian Fluid Mechanics, vol. 73, no. 1-2, pp. 163-179, 1997.
[246] J. R. Macdonald, "On relaxation-spectrum estimation for decades of data: accuracy and sampling-localization considerations," Inverse Problems, vol. 16, no. 5, pp. 1561-1583, 2000.

[247] M. Baumgaertel and H. H. Winter, "Determination of discrete relaxation and retardation time spectra from dynamic mechanical data," Rheologica Acta, vol. 28, no. 6, pp. 511-519, 1989.

[248] S. Hansen, "Estimation of the relaxation spectrum from dynamic experiments using Bayesian analysis and a new regularization constraint," Rheologica Acta, vol. 47, no. 2, pp. 169-178, 2008.

[249] S. W. Provencher, "An eigenfunction expansion method for the analysis of exponential decay curves," The Journal of Chemical Physics, vol. 64, no. 7, pp. 2772-2777, 1976.

[250] S. W. Provencher, "CONTIN: a general purpose constrained regularization program for inverting noisy linear algebraic and integral equations," Computer Physics Communications, vol. 27, no. 3, pp. 229-242, 1982.

[251] J. Weese, "A reliable and fast method for the solution of Fredhol integral equations of the first kind based on Tikhonov regularization," Computer Physics Communications, vol. 69, no. 1, pp. 99-111, 1992.

[252] J. Honerkamp and J. Weese, "A nonlinear regularization method for the calculation of relaxation spectra," Rheologica Acta, vol. 32, no. 1, pp. 65-73, 1993.

[253] J. Weese, "A regularization method for nonlinear ill-posed problems," Computer Physics Communications, vol. 77, no. 3, pp. 429-440, 1993.

[254] T. Roths, M. Marth, J. Weese, and J. Honerkamp, "A generalized regularization method for nonlinear ill-posed problems enhanced for nonlinear regularization terms," Computer Physics Communications, vol. 139, no. 3, pp. 279-296, 2001.

[255] J. Honerkamp and J. Weese, "Tikhonovs regularization method for ill-posed problems-a comparison of different methods for the determination of the regularization parameter," Continuum Mechanics and Thermodynamics, vol. 2, no. 1, pp. 17-30, 1990.

[256] C. W. Groetsch, The Theory of Tikhonov Regularization for Fredholm Equations of the First Kind, Pitman, London, UK, 1984.

[257] S. H. Wasserman, "Calculating the molecular weight distribution from linear viscoelastic response of polymer melts," Journal of Rheology, vol. 39, no. 3, pp. 601-625, 1995.

[258] F. Léonardi, A. Allal, and G. Marin, "Determination of the molecular weight distribution of linear polymers by inversion of a blending law on complex viscosities," Rheologica Acta, vol. 37, no. 3, pp. 199-213, 1998.

[259] D. H. S. Ramkumar and J. M. Wiest, "Molecular weight distributions from linear viscoelastic measurements," Rheologica Acta, vol. 35, no. 4, pp. 356-363, 1996.

[260] F. Cocchini and M. R. Nobile, "Constrained inversion of rheological data to molecular weight distribution for polymer melts," Rheologica Acta, vol. 42, no. 3, pp. 232-242, 2003.

[261] C. Carrot and J. Guillet, "From dynamic moduli to molecular weight distribution: a study of various polydisperse linear polymers," Journal of Rheology, vol. 41, no. 5, pp. 1203-1220, 1997.

[262] M. R. Nobile and F. Cocchini, "Evaluation of molecular weight distribution from dynamic moduli," Rheologica Acta, vol. 40, no. 2, pp. 111-119, 2001.

[263] C. Pattamaprom, R. G. Larson, and A. Sirivat, "Determining polymer molecular weight distributions from rheological 
properties using the dual-constraint model," Rheologica Acta, vol. 47, no. 7, pp. 689-700, 2008.

[264] C. Pattamaprom, R. G. Larson, and T. J. van Dyke, "Quantitative predictions of linear viscoelastic rheological properties of entangled polymers," Rheologica Acta, vol. 39, no. 6, pp. 517-531, 2000.

[265] R. S. Anderssen, D. W. Mead, and J. J. Driscoll IV, "On the recovery of molecular weight functionals from the double reptation model," Journal of Non-Newtonian Fluid Mechanics, vol. 68, no. 2-3, pp. 291-301, 1997.

[266] I. N. Sneddon, The Use of Integral Transforms, McGraw-Hill, New York, NY, USA, 1972.

[267] R. Bracewell, The Fourier Transform and Its Applications, McGraw-Hill, New York, NY, USA, 2000.

[268] M. T. Heath, Scientific Computing: An Introductory Survey, McGraw-Hill, New York, NY, USA, 2nd edition, 2002.

[269] R. L. Wolpert and K. Ickstadt, "Reflecting uncertainty in inverse problems: a Bayesian solution using Lévy processes," Inverse Problems, vol. 20, no. 6, pp. 1759-1771, 2004.

[270] J. D. Guzmán, J. D. Schieber, and R. Pollard, "A regularization-free method for the calculation of molecular weight distributions from dynamic moduli data," Rheologica Acta, vol. 44, no. 4, pp. 342-351, 2005.

[271] M. Doi, "Basic principle in the viscoelasticity of polymeric liquids," Journal of Non-Newtonian Fluid Mechanics, vol. 23, pp. 151-162, 1987.

[272] F. Léonardi, A. Allal, and G. Marin, "Molecular weight distribution from viscoelastic data: the importance of tube renewal and rouse modes," Journal of Rheology, vol. 46, no. 1, pp. 209-224, 2002.

[273] J. M. Dealy, "Letter to the editor: comment on "on the rouse spectrum and the determination of the molecular weight distribution from rheological data"', Journal of Rheology, vol. 45, no. 2, pp. 603-604, 2001.

[274] P. J. Doerpinghaus and D. G. Baird, "Separating the effects of sparse long-chain branching on rheology from those due to molecular weight in polyethylenes," Journal of Rheology, vol. 47, no. 3, pp. 717-736, 2003.

[275] H. M. Laun and H. Schuch, "Transient elongational viscosities and drawability of polymer melts," Journal of Rheology, vol. 33, no. 1, pp. 119-175, 1989.

[276] J. F. Vega, A. Santamaría, A. Muñoz-Escalona, and P. Lafuente, "Small-amplitude oscillatory shear flow measurements as a tool to detect very low amounts of long chain branching in polyethylenes," Macromolecules, vol. 31, no. 11, pp. 3639-3647, 1998.

[277] C. Piel, F. J. Stadler, J. Kaschta, S. Rulhoff, H. Münstedt, and W. Kaminsky, "Structure-property relationships of linear and long-chain branched metallocene high-density polyethylenes characterized by shear rheology and SEC-MALLS," Macromolecular Chemistry and Physics, vol. 207, no. 1, pp. 26-38, 2006.

[278] R. N. Shroff and H. Mavridis, "Long-chain-branching index for essentially linear polyethylenes," Macromolecules, vol. 32, no. 25, pp. 8454-8464, 1999.

[279] W. J. Wang, S. Kharchenko, K. Migler, and S. Zhu, "Tripledetector GPC characterization and processing behavior of long-chain-branched polyethylene prepared by solution polymerization with constrained geometry catalyst," Polymer, vol. 45, no. 19, pp. 6495-6505, 2004.

[280] J. F. Vega, M. Fernández, A. Santamaria, A. Muñoz-Escalona, and P. Lafuente, "Rheological criteria to characterize metallocene catalyzed polyethylenes," Macromolecular Chemistry and Physics, vol. 200, no. 10, pp. 2257-2268, 1999.
[281] W. Thimm, C. Friedrich, T. Roths, S. Trinkle, and J. Honerkamp, "Characterization of long-chain branching effects in linear rheology," arXiv:cond-mat/0009169v1, 2000.

[282] E. van Ruymbeke, S. Coppola, L. Balacca, S. Righi, and D. Vlassopoulos, "Decoding the viscoelastic response of polydisperse star/linear polymer blends," Journal of Rheology, vol. 54, no. 3, pp. 507-538, 2010.

[283] H. P. Schreiber and E. B. Bagley, "The Newtonian melt viscosity of polyethylene: an index of long-chain branching," Journal of Polymer Science A, vol. 58, no. 166, pp. 29-48, 1962.

[284] S. Lai, T. A. Plumley, T. I. Butler, G. W. Knight, and C. I. Kao, "Dow rheology index (DRI) for insite technology polyolefins (ITP): unique structure-processing relationships," in Proceedings of the 52nd Annual Technical Conference (ANTEC '94), vol. 40, p. 1814, May 1994.

[285] J. C. Michel, "Introduction of a new rheological long chain branching index for isotactic polypropylene melts," in Proceedings of the Annual Technical Conference (ANTEC '02), pp. 1-5, 2002.

[286] C. J. Tsenoglou and A. D. Gotsis, "Rheological characterization of long chain branching in a melt of evolving molecular architecture," Macromolecules, vol. 34, no. 14, pp. 4685-4687, 2001.

[287] C. Gabriel and H. Münstedt, "Creep recovery behavior of metallocene linear low-density polyethylenes," Rheologica Acta, vol. 38, no. 5, pp. 393-403, 1999.

[288] C. Gabriel, E. Kokko, B. Löfgren, J. Seppälä, and H. Münstedt, "Analytical and rheological characterization of long-chain branched metallocene-catalyzed ethylene homopolymers," Polymer, vol. 43, no. 24, pp. 6383-6390, 2002.

[289] C. Gabriel and H. Münstedt, "Influence of long-chain branches in polyethylenes on linear viscoelastic flow properties in shear," Rheologica Acta, vol. 41, no. 3, pp. 232-244, 2002.

[290] D. J. Plazek and I. Echeverría, "Don't cry for me Charlie Brown, or with compliance comes comprehension," Journal of Rheology, vol. 44, no. 4, pp. 831-841, 2000.

[291] F. J. Stadler, J. Kaschta, and H. Münstedt, "Thermorheological behavior of various long-chain branched polyethylenes," Macromolecules, vol. 41, no. 4, pp. 1328-1333, 2008.

[292] F. J. Stadler and H. Münstedt, "Numerical description of shear viscosity functions of long-chain branched metallocene-catalyzed polyethylenes," Journal of Non-Newtonian Fluid Mechanics, vol. 151, no. 1-3, pp. 129-135, 2008.

[293] F. J. Stadler, D. Auhl, and H. Münstedt, "Influence of the molecular structure of polyolefins on the damping function in shear," Macromolecules, vol. 41, no. 10, pp. 3720-3726, 2008.

[294] P. M. Wood-Adams, "The effect of long chain branches on the shear flow behavior of polyethylene," Journal of Rheology, vol. 45, no. 1, pp. 203-210, 2001.

[295] G. V. Vinogradov and A. Malkin, "Rheological properties of polymer melts," Journal of Polymer Science B, vol. 4, no. 1, pp. 135-154, 1966.

[296] F. J. Stadler, C. Piel, K. Klimke et al., "Influence of type and content of various comonomers on long-chain branching of ethene/ $\alpha$-olefin copolymers," Macromolecules, vol. 39, no. 4, pp. 1474-1482, 2006.

[297] S. Trinkle, P. Walter, and C. Friedrich, "Van Gurp-Palmen plot II - Classification of long chain branched polymers by their topology," Rheologica Acta, vol. 41, no. 1, pp. 103-113, 2002.

[298] M. van Gurp and J. Palmen, "Time-temperature superposition for polymeric blends," Rheology Bulletin, vol. 67, pp. 5-8, 1998. 
[299] J. Liu, W. Yu, and C. Zhou, "Polymer chain topological map as determined by linear viscoelasticity," Journal of Rheology, vol. 55, no. 3, pp. 545-570, 2011.

[300] S. Trinkle and C. Freidrich, "Van Gurp-Palmen-plot: a way to characterize polydispersity of linear polymers," Rheologica Acta, vol. 40, no. 4, pp. 322-328, 2001.

[301] C. A. García-Franco, S. Srinivas, D. J. Lohse, and P. Brant, "Similarities between gelation and long chain branching viscoelastic behavior," Macromolecules, vol. 34, no. 10, pp. 3115-3117, 2001.

[302] C. G. Robertson, C. A. García-Franco, and S. Srinivas, "Extent of branching from linear viscoelasticity of longchain-branched polymers," Journal of Polymer Science B, vol. 42, no. 9, pp. 1671-1684, 2004.

[303] J. A. Resch, U. Keßner, and F. J. Stadler, “Thermorheological behavior of polyethylene: a sensitive probe to molecular structure," Rheologica Acta, vol. 50, no. 5-6, pp. 559-575, 2011.

[304] P. Wood-Adams and S. Costeux, "Thermorheological behavior of polyethylene: effects of microstructure and long chain branching," Macromolecules, vol. 34, no. 18, pp. 6281-6290, 2001.

[305] B. H. Bersted, "On the effects of very low levels of long chain branching on rheological behavior in polyethylene," Journal of Applied Polymer Science, vol. 30, no. 9, pp. 3751-3765, 1985.

[306] E. Kolodka, W. J. Wang, S. Zhu, and A. Hamielec, "Rheological and thermomechanical properties of long-chainbranched polyethylene prepared by slurry polymerization with metallocene catalysts," Journal of Applied Polymer Science, vol. 92, no. 1, pp. 307-316, 2004.

[307] J. Meissner, "Rheometer for the study of mechanical properties of deformation of plastic melts under definite tensile stress," Rheologica Acta, vol. 8, no. 1, pp. 78-88, 1969.

[308] R. G. Larson, "Nonlinear shear relaxation modulus for a linear lowdensity polyethylene," Journal of Rheology, vol. 29, no. 6, pp. 823-831, 1985.

[309] T. Neidhöfer, S. Sioula, N. Hadjichristidis, and M. Wilhelm, "Distinguishing linear from star-branched polystyrene solutions with Fourier-transform rheology," Macromolecular Rapid Communications, vol. 25, no. 22, pp. 1921-1926, 2004.

[310] L. A. Archer and S. K. Varshney, "Synthesis and relaxation dynamics of multiarm polybutadiene melts," Macromolecules, vol. 31, no. 18, pp. 6348-6355, 1998.

[311] J. Hepperle and H. Münstedt, "Rheological properties of branched polystyrenes: nonlinear shear and extensional behavior," Rheologica Acta, vol. 45, no. 5, pp. 717-727, 2006.

[312] F. J. Stadler, A. Nishioka, J. Stange, K. Koyama, and H. Münstedt, "Comparison of the elongational behavior of various polyolefins in uniaxial and equibiaxial flows," Rheologica Acta, vol. 46, no. 7, pp. 1003-1012, 2007.

[313] C. Gabriel and H. Münstedt, "Strain hardening of various polyolefins in uniaxial elongational flow," Journal of Rheology, vol. 47, no. 3, pp. 619-630, 2003.

[314] G. Fleury, G. Schlatter, and R. Muller, "Non Linear Rheology for Long Chain Branching characterization, comparison of two methodologies: fourier transform rheology and relaxation," Rheologica Acta, vol. 44, no. 2, pp. 174-187, 2004.

[315] F. J. Stadler and H. Münstedt, "Correlations between the shape of viscosity functions and the molecular structure of long-chain branched polyethylenes," Macromolecular Materials and Engineering, vol. 294, no. 1, pp. 25-34, 2009.

[316] P. J. Doerpinghaus and D. G. Baird, "Assessing the branching architecture of sparsely branched metallocene-catalyzed polyethylenes using the pompom constitutive model," Macromolecules, vol. 35, no. 27, pp. 10087-10095, 2002.

[317] H. C. Öttinger, "Thermodynamic admissibility of the pompon model for branched polymers," Rheologica Acta, vol. 40, no. 4, pp. 317-321, 2001.

[318] N. J. Inkson and T. N. Phillips, "Unphysical phenomena associated with the extended pom-pom model in steady flow," Journal of Non-Newtonian Fluid Mechanics, vol. 145, no. 2-3, pp. 92-101, 2007.

[319] G. Bishko, T. C. B. McLeish, O. G. Harlen, and R. G. Larson, "Theoretical molecular rheology of branched polymers in simple and complex flows: the Pom-Pom model," Physical Review Letters, vol. 79, no. 12, pp. 2352-2355, 1997.

[320] P. Rubio and M. H. Wagner, "LDPE melt rheology and the pom-pom model," Journal of Non-Newtonian Fluid Mechanics, vol. 92, no. 2-3, pp. 245-259, 2000.

[321] M. Wilhelm, D. Maring, and H. W. Spiess, "Fourier-transform rheology," Rheologica Acta, vol. 37, no. 4, pp. 399-405, 1998.

[322] M. Wilhelm, P. Reinheimer, and M. Ortseifer, "High sensitivity Fourier-transform rheology," Rheologica Acta, vol. 38, no. 4, pp. 349-356, 1999.

[323] M. Wilhelm, "Fourier-transform rheology," Macromolecular Materials and Engineering, vol. 287, no. 2, pp. 83-105, 2002.

[324] M. Wilhelm, P. Reinheimer, M. Ortseifer, T. Neidhöpofer, and H. W. Spiess, "The crossover between linear and non-linear mechanical behaviour in polymer solutions as detected by Fourier-transform rheology," Rheologica Acta, vol. 39, no. 3, pp. 241-246, 2000.

[325] T. Neidhöpofer, M. Wilhelm, and H. W. Spiess, "Fouriertransformrheology on linear polystyrene melts," Applied Rheology, vol. 11, pp. 126-133, 2001.

[326] K. Hyun, K. H. Ahn, S. J. Lee, M. Sugimoto, and K. Koyama, "Degree of branching of polypropylene measured from Fourier-transform rheology," Rheologica Acta, vol. 46, no. 1, pp. 123-129, 2006.

[327] I. Vittorias, M. Parkinson, K. Klimke, B. Debbaut, and M. Wilhelm, "Detection and quantification of industrial polyethylene branching topologies via Fourier-transform rheology, NMR and simulation using the Pom-pom model," Rheologica Acta, vol. 46, no. 3, pp. 321-340, 2007.

[328] M. Ansari, S. G. Hatzikiriakos, A. M. Sukhadia, and D. C. Rohlfing, "Rheology of Ziegler-Natta and metallocene highdensity polyethylenes: broad molecular weight distribution effects," Rheologica Acta, vol. 50, no. 1, pp. 17-27, 2011.

[329] C. P. Lusignan, T. H. Mourey, J. C. Wilson, and R. H. Colby, "Viscoelasticity of randomly branched polymers in the critical percolation class," Physical Review E, vol. 52, no. 6, pp. 6271-6280, 1995.

[330] C. P. Lusignan, T. H. Mourey, J. C. Wilson, and R. H. Colby, "Viscoelasticity of randomly branched polymers in the vulcanization class," Physical Review E, vol. 60, no. 5, pp. 5657-5669, 1999.

[331] C. He, S. Costeux, and P. Wood-Adams, "A technique to infer structural information for low level long chain branched polyethylenes," Polymer, vol. 45, no. 11, pp. 3747-3754, 2004.

[332] M. T. Shaw and W. H. Tuminello, "Closer look at the MWDviscosity transform," Polymer Engineering \& Science, vol. 34, no. 2, pp. 159-165, 1994.

[333] B. J. Crosby, M. Mangnus, W. de Groot, R. Daniels, and T. C. B. McLeish, "Characterization of long chain branching: dilution rheology of industrial polyethylenes," Journal of Rheology, vol. 46, no. 2, pp. 401-426, 2002. 
[334] B. Krause, D. Voigt, A. Lederer, D. Auhl, and H. Münstedt, "Determination of low amounts of long-chain branches in polypropylene using a combination of chromatographic and rheological methods," Journal of Chromatography A, vol. 1056, no. 1, pp. 217-222, 2004.

[335] F. Stadler, "Detecting very low levels of long-chain branching in metallocene-catalyzed polyethylenes," Rheologica Acta, vol. 51, no. 9, pp. 821-840, 2012.

[336] P. Castignolles, R. Graf, M. Parkinson, M. Wilhelm, and M. Gaborieau, "Detection and quantification of branching in polyacrylates by size-exclusion chromatography (SEC) and melt-state ${ }^{13} \mathrm{C}$ NMR spectroscopy," Polymer, vol. 50, no. 11, pp. 2373-2383, 2009.

[337] D. J. Read and T. C. B. McLeish, "Molecular rheology and statistics of long chain branched metallocene-catalyzed polyolefins," Macromolecules, vol. 34, no. 6, pp. 1928-1945, 2001.

[338] J. B. P. Soares and A. E. Hamielec, "Bivariate chain length and long chain branching distribution for copolymerization of olefins and polyolefin chains containing terminal doublebonds," Macromolecular Theory and Simulations, vol. 5, no. 3, pp. 547-572, 1996.

[339] H. Tobita and A. E. Hamielec, "Control of network structure in free-radical crosslinking copolymerization," Polymer, vol. 33, no. 17, pp. 3647-3657, 1992.

[340] H. Tobita, "Cross-linking kinetics in emulsion copolymerization," Macromolecules, vol. 25, no. 10, pp. 2671-2678, 1992.

[341] H. Tobita, "Molecular weight distribution in free-radical cross-linking copolymerization," Macromolecules, vol. 26, no. 4, pp. 836-841, 1993.

[342] H. Tobita and K. Yamamoto, "Network formation in emulsion cross-linking copolymerization," Macromolecules, vol. 27, no. 12, pp. 3389-3396, 1994.

[343] H. Tobita, "Simultaneous long-chain branching and random scission: I. Monte Carlo simulation," Journal of Polymer Science B, vol. 39, no. 4, pp. 391-403, 2001.

[344] H. Tobita, "Molecular weight distribution of living radical polymers," Macromolecular Theory and Simulations, vol. 15, no. 1, pp. 12-22, 2006.

[345] J. Stange, C. Uhl, and H. Münstedt, "Rheological behavior of blends from a linear and a long-chain branched polypropylene," Journal of Rheology, vol. 49, no. 5, pp. 1059-1079, 2005.

[346] S. Shanbhag, "Analytical rheology of blends of linear and star polymers using a Bayesian formulation," Rheologica Acta, vol. 49, no. 4, pp. 411-422, 2010.

[347] S. Shanbhag, "Analytical rheology of branched polymer melts: identifying and resolving degenerate structures," Journal of Rheology, vol. 55, no. 1, pp. 177-194, 2011.

[348] A. Takeh, J. Worch, and S. Shanbhag, "Analytical rheology of metallocene-catalyzed polyethylenes," Macromolecules, vol. 44, no. 9, pp. 3656-3665, 2011.

[349] A. Tarantola, Inverse Problem Theory and Methods for Model Parameter Estimation, Society for Industrial and Applied Mathematics, Philadelphia, Pa, USA, 2005.

[350] R. S. B. Anderssen, "Inverse problems: a pragmatist's approach to the recovery of information from indirect measurements," ANZIAM Journal, vol. 46, no. 5, pp. C588-C622, 2004.

[351] P. J. Green, "Reversible jump Markov chain monte carlo computation and Bayesian model determination," Biometrika, vol. 82, no. 4, pp. 711-732, 1995.

[352] C. D. MacKay, Bayesian methods for adaptive modelling [Ph.D. thesis], California Institute of Technology, 1991.
[353] I. Murray and I. M. Z. Ghahramani, "A note on the evidence and Bayesian Occam's razor," Gatsby Computational Neuroscience Unit Tech Report, pp. 1-4, 2005. 

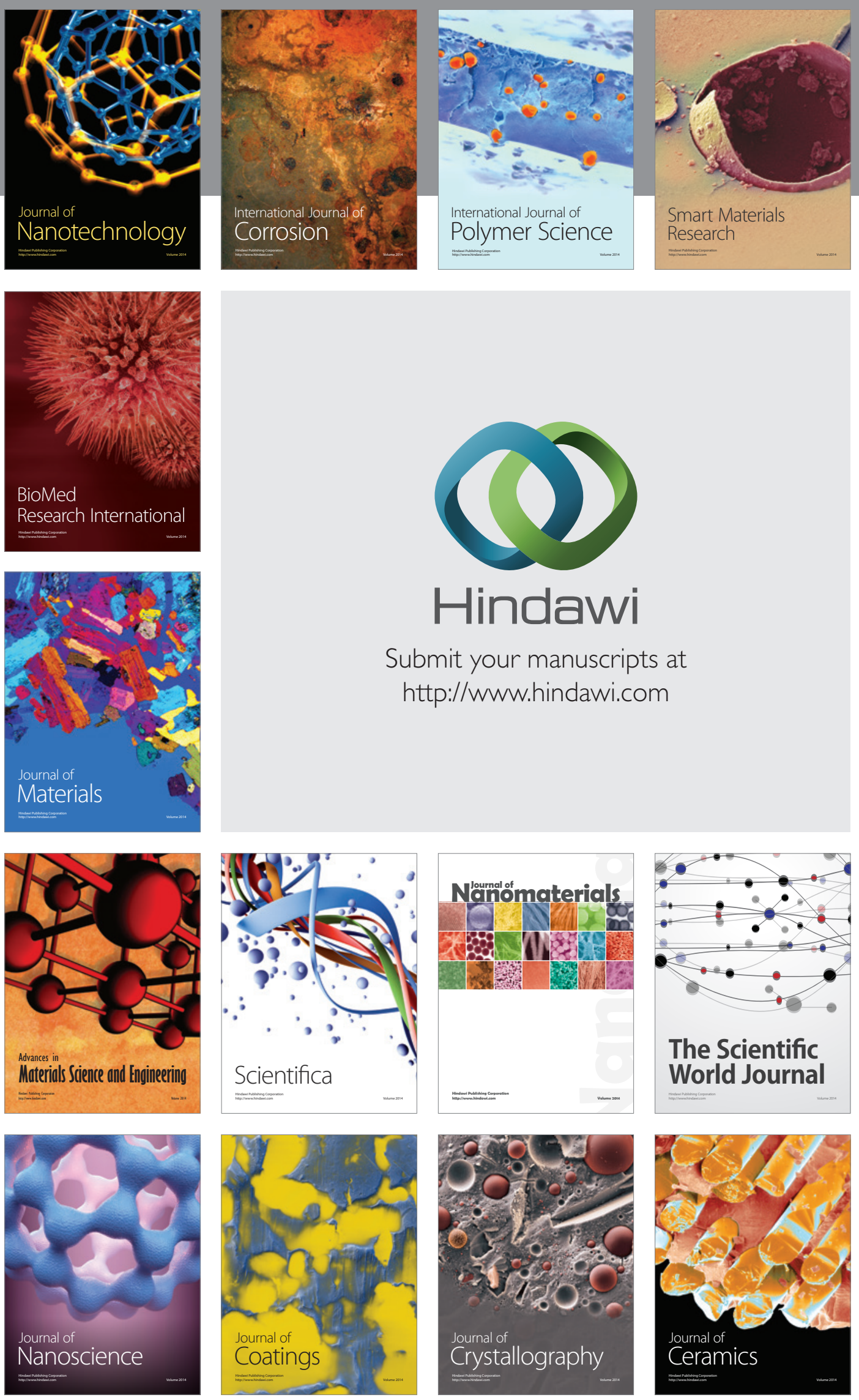

The Scientific World Journal

Submit your manuscripts at

http://www.hindawi.com

\section{World Journal}

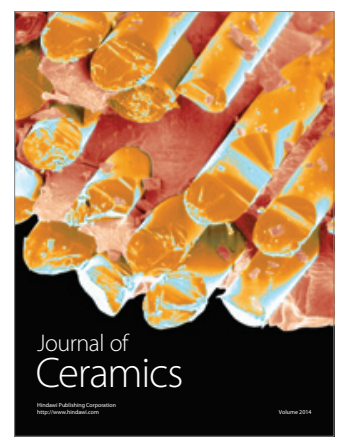

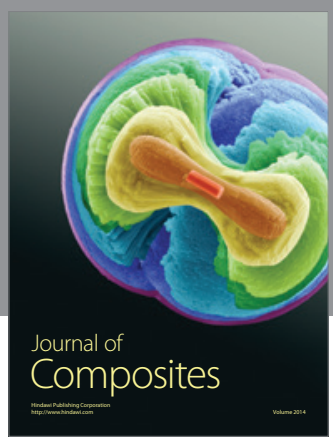
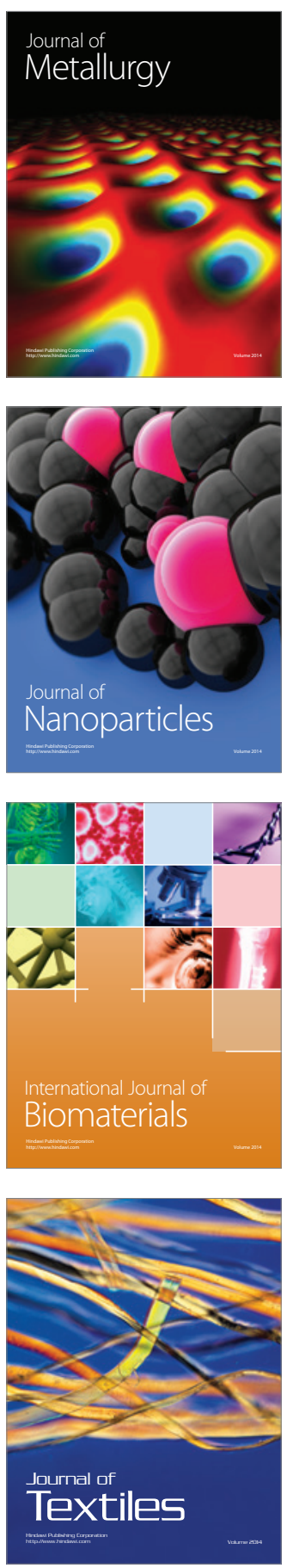